Japan. J. Math.

Vol. 10, No. 2, 1984

\title{
A complement to the theory of $G$-CW complexes
}

\author{
Takao Matumoto \\ (Received October 1, 1984)
}

\section{Introduction}

Let $G$ be a topological group. By a $G$-space $X$ we mean a topological space $X$ together with a continuous $G$-action on $X$. If a $G$-map (i.e. $G$ equivariant continuous map) $f: X \rightarrow Y$ between $G$-spaces induces isomorphisms $f_{*}: \pi_{n}\left(X^{H}, x\right) \rightarrow \pi_{n}\left(X^{H}, f(x)\right)$ for every integer $n \geqq 0$, every subgroup $H$ of $G$ and any $x \in X^{H}=\{x \in X$; $g x=x$ for every $g \in H\}$, then $f$ is called a weak $G$-homotopy equivalence. We have defined in [5] and [6] the notion of $G$-CW complexes and have shown that any weak $G$-homotopy equivalence between $G$-CW complexes is a $G$-homotopy equivalence. Note that, if $X$ is a Hausdorff space, $X^{H}=X^{\bar{H}}$ for the closure $\bar{H}$ of $H$.

The purpose of this paper is to construct in a canonical way a $G$-CW complex $K_{X}$ and a weak $G$-homotopy equivalence $\rho_{X}: K_{X} \rightarrow X$ for an arbitrary $G$-space $X$ which satisfies the $T_{1}$-separability condition (Theorem 1). When $X$ is not a $T_{1}$-space, the same construction gives us a $K_{X}$ which is a $G$-CW complex in the wider sense that the underlying space is not necessarily a Hausdorff space. We call this simply a $G$-CW complex hereafter in this paper: and a $G$-CW complex in the sense of [6] will be called a Hausdorff $G$-CW complex. We presume that there will be no serious confusion in the future because there still hold the $G$-cellular approximation theorem, the $G$-homotopy extension property, the theorem of J.H.C. Whitehead type and so on for $G$-CW complexes in this paper; in addition, there is a simple criterion which says that a $G$-CW complex is a Hausdorff $G$-CW complex if and only if the isotropy subgroups are closed subgroups of $G$ (Proposition $0.1)$.

As an application we prove that $\rho_{X}: K_{X} \rightarrow X$ induces an isomorphism for the singular $G$-(co) homology theory defined by Illman [4] (Theorems 2 and $\left.2^{*}\right)$. Since we have a strongly convergent spectral sequence of AtiyahHirzebruch type (Proposition 3.1), the singular $G$-(co) homology theory with coefficient in a generic $G$-coefficient system on $X$ coincides with the cellular $G$-(co) homology theory on $K_{X}$ (Theorem 3).

The other applications of Theorem 1 are found in Murayama [11] and 
Waner [13]. For example, if $G$ is a compact Lie group, then a G-ANR has the $G$-homotopy type of a $G$-CW complex. Since $G L(G)$ is a $G$-ANR, this gives a schematic proof of the main theorem of [5]. We shall discuss the possibility of another construction of the $G$-homotopy type of $K_{X}$ in the last. part of $\S 1$.

The results were announced in RIMS Kokyuroku 419 (1981), 26-27. The author would like to thank Dr. M. Murayama who has helped him in writing up this paper since then. He expresses his gratitude also to Professor M. Sugawara for his interest and, in particular, for an improvement of the proof of Theorems 2 and $2^{*}$.

\section{$\S 0 . \quad G$-CW complexes and Hausdorff $G$-CW complexes}

Let $G$ be a not necessarily Hausdorff topological group. We shall extend the theory of $G$-CW complexes of [6] to the case of non-Hausdorff $G$-spaces whose orbit spaces are Hausdorff. Let $\Delta^{n}$ denote the geometric $n$-simplex $\left\{t=\left(t_{0}, \cdots, t_{n}\right) \in R^{n+1} ; 0 \leqq t_{i} \leqq 1, \sum t_{i}=1\right\}$ with the trivial $G$-action and $\partial \Delta^{n}$ its boundary. If $f: A \rightarrow Y$ is a $G$-map defined on a closed $G$-invariant subspace $A$ of a $G$-space $X$ to another $G$-space $Y$, then the adjunction space $Y \bigcup_{f} X=(Y \amalg X) / \sim$, where $f(x) \sim x(x \in A)$, is naturally a $G$-space.

Definition. Let $X^{n}(n \geqq 0)$ be the $G$-space defined inductively by

$$
X^{0}=\prod_{\lambda \in \Lambda_{0}} G / G_{\lambda} \times \Delta^{0} \quad \text { and } \quad X^{n}=X^{n-1} \bigcup_{\psi_{n}}\left(\coprod_{\lambda \in A_{n}} G / G_{\lambda} \times \Delta^{n}\right)
$$

for a family of subgroups $G_{\lambda}$ of $G$ and some $G$-maps $\psi_{n}$ : $G / G_{\lambda} \times \partial \Delta^{n} \rightarrow X^{n-1}$, where $G$ acts on $\Delta^{n}$ trivially. Then, the direct limit $X=\cup X^{n}$ has the structure of a $G$-space so that it contains $X^{n}$ as a closed $G$-subspace for any $n$. We call a $G$-space $X=\cup X^{n}$ with a decomposition defined above a $G$-CW complex.

For each $\lambda \in \Lambda_{n}$, there is a natural characteristic $G$-map $G \sigma_{\lambda}: G / G_{\lambda} \times \Delta^{n}$ $\rightarrow X^{n} \subset X$. We define $e_{\lambda}=G \sigma_{\lambda}\left(G_{\lambda} / G_{\lambda} \times\left(\Delta^{n}-\partial \Delta^{n}\right)\right)$, and call its $G$-orbit $G e_{\lambda}$ an $n$-dimensional $G$-cell or simply a $G$ - $n$-cell, and $G_{\lambda}$ the isotropy subgroup at $G e_{\lambda}$ or at $e_{\lambda}$. When we write $G \sigma=G \sigma_{\lambda}, G_{\lambda}$ is denoted by $G_{\sigma}$ and the image of $G \sigma$, called a closed $G$-cell, is also denoted by the same symbol $G \sigma$. When we write $e=e_{\lambda}, G_{\lambda}$ and $G \sigma_{\lambda}$ will be denoted by $G_{e}$ and $G \sigma_{e}$, respectively. Then, the image of $G \sigma_{e}: G / G_{e} \times \Delta^{n} \rightarrow X^{n} \subset X$ is the closure $\overline{G e}$ of $G e$ and

(b1) $G \sigma_{e} \mid G / G_{e} \times\left(\Delta^{n}-\partial \Delta^{n}\right)$ is a homeomorphism onto $G e$, and

(b2) $\partial G e=\overline{G e}-G e=G \partial e=G \sigma_{e}\left(G / G_{e} \times \partial \Delta^{n}\right)$ is contained in $X^{n-1}$.

REMARK. On the orbit spaces, $X^{0} / G$ is a discrete space and $X^{n} / G$ is obtained from $X^{n-1} / G$ by attaching the $n$-cells $\Delta^{n}$ by $p r \circ\left(\sigma_{\lambda} \mid \partial \Delta^{n}\right): \partial \Delta^{n} \rightarrow X^{n-1}$ 
$\rightarrow X^{n-1} / G$ for $\lambda \in \Lambda_{n}$, where $\sigma_{\lambda}=G \sigma_{\lambda} \mid G_{\lambda} / G_{\lambda} \times \Delta^{n}$ and $p r$ denotes the projection. So, $X / G=\cup X^{n} / G$ is a $C W$-complex and, in particular, a Hausdorff space.

We note here an equivalent definition of $G$-CW complexes.

Definition. Let $X$ be a $G$-space whose orbit space $X / G$ is a Hausdorff space. We say that a family $G K=\{G e \subset X\}$ determines a $G$-cell complex structure on $X$ if:

(a) $X=\bigcup_{G e \in G K} G e$ (disjoint union), and

(b) each $G e=G e^{n} \in G K$, called a $G$ - $n$-cell, has its characteristic $G$-map onto its closure, $G \sigma_{e}: G / G_{e} \times \Delta^{n} \rightarrow \overline{G e} \subset X$, for some subgroup $G_{e}$ of $G$, satisfying (b1) and (b2) where $X^{n-1}$ is the union of all $G$-m-cells with $m \leqq n-1$.

When $X$ is a Hausdorff space in addition, the pair $(X, G K)$ in the above definition is called a $G$-cell complex in [6]. Our new definition involves also the case when $X$ is not a Hausdorff space, although it is not necessarily a cell complex in the sense of J.H.C. Whitehead. Notice that we used the Hausdorff property of $X$ only to show that the closure of $e=G \sigma_{e}\left(G_{e} / G_{e} \times\right.$ $\left.\left(\Delta^{n}-\partial \Delta^{n}\right)\right)$ coincides with $\tilde{e}=G \sigma_{e}\left(G_{e} / G_{e} \times \Delta^{n}\right)$. We have $\overline{G e}=G \tilde{e}$ in our case by the Hausdorff property of $X / G$, and $G e / G$ is a cell of $X / G$. Hence, a $G$ cell complex structure on $X$ induces a cell complex structure on $X / G$.

Definition. A $G$-cell complex $(X, G K)$ is called a $G$-CW complex if it satisfies the following two conditions:

(G-C) $G$-closure finiteness, that is, the induced cell complex structure on the orbit space $X / G$ is closure finite.

$(G$-W) $G$-weak topology, that is, $X$ has the weak topology with respect to the closed covering $\{\overline{G e} ; G e \in G K\}$ of $X$.

The equivalence of the above two definitions of a $G$-CW complex is obvious.

The relation between $G$-CW complexes and Hausdorff $G$-CW complexes is given in the following proposition which I owe to M. Murayama.

Proposition 0.1. Let $X$ be a G-CW complex in the sense of this paper. Then, the following three conditions are equivalent to each other:

(1) $X$ is a Hausdorff space and hence a Hausdorff $G$-CW complex ( $a$ G$C W$ complex in the sense of [6]).

(2) $G / G_{e}$ is a Hausdorff space for every G-cell Ge in $X$.

(3) The isotropy subgroup $G_{e}$ is a closed subgroup of $G$ for every G-cell Ge in $X$.

Proof. The implications $(1) \Rightarrow(2)$ and $(2) \Rightarrow(3)$ are immediate. The 
implication $(3) \Rightarrow(2)$ is not difficult and shown in [10, p, 27]. So, we have only to prove that (2) implies (1). Let $x$ and $y$ be distinct points of $X$. We should find open subsets $U_{x}$ and $U_{y}$ such that $U_{x} \ni x, U_{y} \ni y$ and $U_{x} \cap U_{y}=\phi$. When $G x \neq G y$, it is easy to see it by the Hausdorff property of $X / G$. So, we may assume that $G x=G y$. A retraction $r: U \rightarrow G x$ of an open neighborhood of $G x$ is constructed as follows. Let $G e$ be a unique (open) $G$ - $n$-cell which contains $x$. Since $G e$ is homeomorphic to $G / G_{e} \times\left(\Delta^{n}-\partial \Delta^{n}\right)$, we have a retraction $r_{n}: U_{n} \rightarrow G x$ for $U_{n}=G e$. Let $b$ be the barycenter of $\Delta^{n+k}$ and let

$$
\hat{X}^{n+k}=X^{n+k}-\coprod_{\lambda \in \Lambda_{n+k}}\left(G / G_{\lambda} \times b\right)=X^{n+k-1} \bigcup_{\psi_{n+k}}\left(\coprod_{\lambda \in \Lambda_{n+k}} G / G_{\lambda} \times\left(\Delta^{n+k}-b\right)\right) \subset X .
$$

Then, $\hat{X}^{n+k}$ is an open subspace of $X^{n+k}$ and a natural retraction $\Delta^{n+k}-b \rightarrow \partial \Delta^{n+k}$ induces a retraction $f_{n+k}: \hat{X}^{n+k} \rightarrow X^{n+k}$. We define $U_{n+k}$ and $r_{n+k}: U_{n+k} \rightarrow G x(k \geqq 1)$ inductively by $U_{n+k}=f_{n+k}^{-1}\left(r_{n+k-1}^{-1}(G x)\right)$ and $r_{n+k}=$ $r_{n+k-1} \circ\left(f_{n+k} \mid U_{n+k}\right)$. Then, by the weak topology of $X, U=\cup U_{n+k}$ is an open subspace and $r=\cup r_{n+k}: U \rightarrow G x$ is a retraction. Since $x$ and $y$ are contained in $G x$ which is homeomorphic to the Hausdorff space $G / G_{e}$, there exist open subsets $V_{x}$ and $V_{y}$ of $G x$ such that $V_{x} \ni x, V_{y} \ni y$ and $V_{x} \cap V_{y}=\phi$. It suffices now to take $U_{x}=r^{-1}\left(V_{x}\right)$ and $U_{y}=r^{-1}\left(V_{y}\right)$.

q.e.d.

We list some of the basic properties of $G$-CW complexes and remark that their proofs are completely the same as in the case of $G$-CW complexes in the sense of [6].

Proposition 0.2 (G-cellular Approximation Theorem). Let $f: X \rightarrow Y$ be a G-map between $G$-CW complexes. Then, there is a G-homotopy $f_{t}: X \rightarrow Y$ such that $f_{0}=f$ and $f_{1}$ is G-cellular, that is, $f_{1}\left(X^{n}\right) \subset Y^{n}$ for any $n$. Moreover, if $f$ is G-cellular on a $G$-CW subcomplex $A$, then we may even require $f_{t}=f$ on $A$.

Proposition 0.3. (G-homotopy Extension Property). Let $f_{0}: X \rightarrow Y$ be a G-map of a G-CW complex $X$ to an arbitrary $G$-space $Y$. Let $g_{t}: A \rightarrow Y$ be a G-homotopy of $g_{0}=f_{0} \mid A$, where $A$ is a $G$-CW subcomplex of $X$. Then, there is a G-homotopy $f_{t}: X \rightarrow Y$ of $f_{0}$ such that $f_{t} \mid A=g_{t}$.

Proposition 0.4. (Theorem of J.H.C. Whitehead). Let $f:(X, A) \rightarrow(Y, B)$ be a G-map between two pairs of $G$-CW complexes. Suppose that $f$ is a weak $G$-homotopy equivalence, that is, the induced maps

$$
f_{*}: \pi_{n}\left(X^{H}, x\right) \longrightarrow \pi_{n}\left(Y^{H}, f(x)\right) \quad \text { and } \quad f_{*}: \pi_{n}\left(A^{H}, a\right) \longrightarrow \pi_{n}\left(B^{H}, f(a)\right)
$$

are bijections for any subgroup $H$ of $G$, any $n \geqq 0$, any $x \in X^{H}$ and any $a \in A^{H}$. Then, $f$ is a G-homotopy equivalence. 
Here, we use the convention that $\pi_{0}(\phi)=\phi$. So, the bijectivity of $f_{*}$ implies that $X^{H}$ and $Y^{H}$ are both empty if one of them is empty.

These propositions correspond to Theorem 4.4, (J) and Theorem 5.3*) of [6] respectively.

\section{$\S 1$. Singular $G$-complex of $X$ and construction of $K_{X}$}

Let $X$ be a $G$-space. Then, $G$ acts on the singular complex

$$
S(X)=\cup S_{n}(X), S_{n}(X)=\left\{\sigma: \Delta^{n} \rightarrow X, \text { continuous maps }\right\},
$$

of $X$ by $(g \sigma)(t)=g \sigma(t)$ for $g \in G, \sigma \in S_{n}(X)$ and $t \in \Delta^{n}$, and therefore on its geometric realization

$$
|S(X)|=\coprod S_{n}(X) \times \Delta^{n} / \sim \text {, with }\left(\partial_{i} \sigma, t\right) \sim\left(\sigma, \delta_{i} t\right),\left(s_{i} \sigma, t\right) \sim\left(\sigma, \eta_{i} t\right) .
$$

Here, $\partial_{i} \sigma=\sigma \circ \delta_{i}, s_{i} \sigma=\sigma \circ \eta_{i}$ and $\delta_{i}(t)=\left(t_{0}, \cdots, t_{i-1} \cdot 0, t_{i}, \cdots, t_{n}\right) \in \Delta^{n+1}, \eta_{i}(t)=$ $\left(t_{0}, \cdots, t_{i-1}, t_{i}+t_{i+1}, t_{i+2}, \cdots, t_{n}\right) \in \Delta^{n-1}$ for $t=\left(t_{0}, \cdots, t_{n}\right) \in \Delta^{n}$. In most cases this $G$-action on $|S(X)|$ is not continuous unless $G$ is a discrete group.

We identify the $G$-orbit of $\sigma \in S_{n}(X)$ with the $G$-map $G \sigma: G / G_{\sigma} \times \Delta^{n} \rightarrow X$ given by $G \sigma\left(g G_{\sigma}, t\right)=g \sigma(t)$, where $G_{\sigma}=\{g \in G ; g \sigma=\sigma\}$ is the isotropy subgroup at $\sigma$. Let $S_{G}(X)$ be the family of such $G$-orbits of $S(X) . \quad S_{G}(X)$ may be called the singular $G$-complex of $X$. Define its geometric realization by

$$
\left|S_{G}(X)\right|=\coprod G / G_{\sigma} \times \Delta^{\operatorname{dim} \sigma} / \sim
$$

where the summation ranges over all $G \sigma \in S_{G}(X)$ and the equivalence relation is the same as in $|S(X)|$. Then, the underlying $G$-sets of $|S(X)|$ and $\left|S_{G}(X)\right|$ are identical, the action $G \times\left|S_{G}(X)\right| \rightarrow\left|S_{G}(X)\right|$ and the "identity" map, denoted by ' $i d$ ': $|S(X)| \rightarrow\left|S_{G}(X)\right|$, are continuous. Let

$$
\tilde{r}_{X}=` i d \text { ' } r_{X}:|S(X)| \rightarrow X \text { and } r_{X}:\left|S_{G}(X)\right| \rightarrow X
$$

be the evaluation maps defined by $\tilde{r}_{X}(\sigma, t)=\sigma(t)$. For any $\sigma \in S_{n}(X)$, let

$$
i_{*}(\sigma): \Delta^{n}=\sigma \times \Delta^{n} \rightarrow|S(X)| \rightarrow\left|S_{G}(X)\right| \quad \text { and } \quad i_{*}(G \sigma): G / G_{\sigma} \times \Delta^{n} \rightarrow\left|S_{G}(X)\right|
$$

be the maps defined canonically by restricting the identification maps. Then,

*) The footnote at p. 371 of [5] is inadequate. ${ }^{6 *} \pi_{k}(X, Y)$ vanishes' should read " $\pi_{k}(X, Y, y)$ vanishes for every point $y$ of $Y$ " and also ' $\varphi_{*}:{ }^{*} \pi_{k}(X) \rightarrow{ }^{*} \pi_{k}(Y)$ is bijective or surjective' should read " $\varphi_{*}: \pi_{k}(X, x) \rightarrow \pi_{k}(Y, \varphi(x))$ is bijective or surjective for every point $x$ of $X$ ". Then, all the statements and proofs are true as stated except that we need a modification in the proof of the implication $(2) \Rightarrow(1)$ of Theorem 5.2; See [8] for the exact proof. Note also that Theorem 5.3 depends only on the implication $(1) \Rightarrow(2)$ of Theorem. 5.2 . 


$$
r_{X} \circ i_{*}(\sigma)=\sigma, \quad r_{X} \circ i_{*}(G \sigma)=G \sigma
$$

and $i_{*}(G \sigma)$ is a $G$-map. Furthermore, $\sigma$ is equivalent to a unique nondegenerate singular $m$-simplex $\tau$ with $m \leqq n$ and $G_{\tau}=G_{\sigma}$. Then, the image of $i_{*}(G \sigma)$ coincides with that of

$$
i_{*}(G \tau): G / G_{\tau} \times \Delta^{m} \longrightarrow\left|S_{G}(X)\right|,
$$

and $i_{*}(G \tau)$ restricts to a homeomorphism on $G / G_{z} \times\left(\Delta^{m}-\partial \Delta^{m}\right)$ and is a characteristic $G$-map of a $G$-cell. It is obvious that $\left|S_{G}(X)\right|$ is a $G$-CW complex with these $G$-cells and that $i_{*}(G \sigma)$ is $G$-cellular. When $X$ is a $T_{1}$-space, the isotropy subgroup $G_{x}$ is a closed subgroup and so is $G_{\sigma}=\bigcap_{x \in \sigma\left(\Delta^{n}\right)} G_{x}$ for any singular $n$-simplex; hence $\left|S_{G}(X)\right|$ is a Hausdorff $G$-CW complex by Proposition 0.1. Thus we have proved the following lemma, where we call a $G$-CW complex to be neat when the characteristic $G$-maps for $G$-cells are all $G$-cellular.

Lemma 1.1. $\left|S_{G}(X)\right|$ is a neat $G$-CW complex and the evaluation map $r_{X}:\left|S_{G}(X)\right| \rightarrow X$ is a continuous G-map. Moreover, if $X$ is a $T_{1}$-space, then $\left|S_{G}(X)\right|$ is a Hausdorff $G-C W$ complex.

We remark that $\left|S_{G}(\cdot)\right|$ is a functor and ' $i d$ ' $/ G:|S(X)| / G \rightarrow\left|S_{G}(X)\right| / G$ is a cellular homeomorphism. Moreover, when $G$ is a discrete group, $\left|S_{G}(X)\right|$ has already the weak $G$-homotopy type of $X$.

Lemma 1.2. For any subgroup $H$ of $G, r^{H}=\left.r_{X}|| S_{G}(X)\right|^{H}:\left|S_{G}(X)\right|^{H} \rightarrow X^{H}$ induces a surjection $r_{*}^{H}: \pi_{n}\left(\left|S_{G}(X)\right|^{H}, X\right) \rightarrow \pi_{n}\left(X^{H}, r_{X}(x)\right)$ for every $n \geqq 0$ and any $x \in\left|S_{G}(X)\right|^{H}$.

Proof. Note that there is a natural identification $\left|S\left(X^{H}\right)\right|=|S(X)|^{H}$ and $r^{H} \circ\left(\left.{ }^{\prime} i d^{\prime}|| S(X)\right|^{H}\right):\left|S\left(X^{H}\right)\right|=|S(X)|^{H} \rightarrow\left|S_{G}(X)\right|^{H} \rightarrow X^{H}$ coincides with the evaluation map for $X^{H}$ which is a weak homotopy equivalence. So, $r_{*}^{H}$ is a surjection.

q.e.d.

Definition. When $X$ is a $G$-CW complex, we define $S_{G}^{c}(X)$ by

$$
S_{G}^{c}(X)=\left\{G \sigma \in S_{G}(X) ; G \sigma: G / G_{\sigma} \times \Delta^{n} \rightarrow X \text { is } G \text {-cellular }\right\}
$$

and $\left|S_{G}^{c}(X)\right|$ by the $G$-CW subcomplex of $\left|S_{G}(X)\right|$ spanned by the elements of $S_{G}^{c}(X)$.

When $\sigma: \Delta^{n} \rightarrow X$ is cellular, that is, $G \sigma: G / G_{\sigma} \times \Delta^{n} \rightarrow X$ is $G$-cellular, the images of the canonical maps $i_{*}(\sigma)$ and $i_{*}(G \sigma)$ are contained in $\left|S_{G}^{c}(X)\right|$ and we have

$$
i_{*}(\sigma): \Delta^{n} \rightarrow\left|S_{G}^{c}(X)\right| \quad \text { and } \quad i_{*}(G \sigma): G / G_{\sigma} \times \Delta^{n} \rightarrow\left|S_{G}^{c}(X)\right| \text {. }
$$


Lemma 1.3. Let $X$ be a $G$-CW complex. Then, the natural inclusion $j:\left|S_{G}^{c}(X)\right| \rightarrow\left|S_{G}(X)\right|$ is a G-deformation retract and $r_{X} \circ j:\left|S_{G}^{c}(X)\right| \rightarrow X$ is a $G$ cellular map. Furthermore, when $X$ is neat, we have a canonical inclusion $i_{*}: X \rightarrow\left|S_{G}^{c}(X)\right|$ defined by $i_{*} \circ G \sigma_{e}=i_{*}\left(G \sigma_{e}\right)$ for any characteristic G-maps $G \sigma_{e}: G / G_{e} \times \Delta^{n} \rightarrow X$ for $G$-cells $G e$ of $X$.

Proof. $\quad r_{X} \circ j$ is clearly $G$-cellular by the definition of $\left|S_{G}^{c}(X)\right|$. By the $G$-cellular approximation theorem (Proposition 0.2), there is a $G$-homotopy $r_{t}:\left|S_{G}(X)\right| \rightarrow X(t \in I)$ such that $r_{0}=r_{X}, r_{t}=r_{X} \circ j$ on $\left|S_{G}^{c}(X)\right|$ and $r_{1}$ is a $G$ cellular map. Then, $f_{t}:\left|S_{G}(X)\right| \rightarrow\left|S_{G}^{c}(X)\right|$, defined by $f_{t} \circ i_{*}(G \sigma)=i_{*}\left(r_{t} \circ i_{*}(G \sigma)\right)$ for $G \sigma \in S_{G}(X)$, is a required $G$-deformation retract, because $f_{0}=i d, f_{t} \| S_{G}^{c}(X) \mid$ $=i d$ and $f_{1}\left(\left|S_{G}(X)\right|\right) \subset\left|S_{G}^{c}(X)\right|$. The last half is clear.

q.e.d.

Definition. Let $f: X_{0} \rightarrow X_{1}$ and $g: X_{0} \rightarrow X_{2}$ be $G$-maps. Then, the double mapping cylinder $M(f, g)$ is defined by

$$
X_{1} \cup_{f} X_{0} \times I \cup_{g} X_{2}=X_{1} \amalg X_{0} \times I \amalg X_{2} / \sim
$$

where $f(x) \sim(x, 0)$ and $(x, 1) \sim g(x)$ for $x \in X_{0}$.

Lemma 1.4. Suppose that $X_{0}, X_{1}$ and $X_{2}$ are $G-C W$ complexes and G-maps $f$ and $g$ are G-cellular. Then, $M(f, g)$ is a G-CW complex. Furthermore, if $X_{0}, X_{1}$ and $X_{2}$ are all neat (resp. Hausdorff), then $M(f, g)$ is neat (resp. Hausdorff).

Proof. The $G$-cells of $M(f, g)$ come from the $G$-cells of $X_{1}$, (the $G$-cells of $\left.X_{0}\right) \times I$ and the $G$-cells of $X_{2}$. These $G$-cells form naturally a $G$-CW complex. Hence, if $X_{0}, X_{1}$ and $X_{2}$ are neat, so is $M(f, g)$. If $X_{0}, X_{1}$ and $X_{2}$ are Hausdorff, then the isotropy subgroups at the $G$-cells are closed and hence $M(f, g)$ is a Hausdorff $G$-CW complex by Proposition 0.1. q.e.d.

Now we construct a neat $G$-CW complex $K_{X}$ and a $G$-map $\rho_{X}: K_{X} \rightarrow X$ for a given $G$-space $X$ :

We construct inductively the neat $G$-CW complexes $X_{n}$ and the $G$-maps $\rho_{n}: X_{n} \rightarrow X$ with $X_{n} \subset X_{n+1}$ and $\rho_{n+1} \mid X_{n}=\rho_{n}$, and then put $K_{X}=\cup X_{n}$ and $\rho_{X}=$ $\cup \rho_{n}$. Define

$$
X_{1}=\left|S_{G}(X)\right| \text { and } \rho_{1}=r_{X}: X_{1}=\left|S_{G}(X)\right| \rightarrow X \text {. }
$$

Suppose that $X_{n}$ and $\rho_{n}$ are already defined. Then, by using $j:\left|S_{G}^{c}\left(X_{n}\right)\right| \subset$ $\left|S_{G}\left(X_{n}\right)\right|$ in Lemma 1.3 , we have the $G$-cellular maps:

$\bar{r}_{n}=r_{X_{n}} \circ j: \bar{X}_{n}=\left|S_{G}^{c}\left(X_{n}\right)\right| \rightarrow X_{n}$ and $\bar{\rho}_{n}=\left|S_{G}\left(\rho_{n}\right)\right| \circ j: \bar{X}_{n} \rightarrow\left|S_{G}(X)\right|$. These satisfy $\rho_{n} \circ \bar{r}_{n}=\rho_{1} \circ \bar{\rho}_{n}$ because $\rho_{n} \circ r_{X_{n}}=r_{X} \circ\left|S_{G}\left(\rho_{n}\right)\right|$. So, we can define $X_{n+1}$ and $\rho_{n+1}$ by 


$$
X_{n+1}=M\left(\bar{r}_{n}, \bar{\rho}_{n}\right) \quad \text { and } \quad \rho_{n+1}=\left(\rho_{n} \amalg \rho_{1} \circ \bar{\rho}_{n} \circ p r \amalg \rho_{1}\right) / \sim
$$

where $p r: \bar{X}_{n} \times I \rightarrow \bar{X}_{n}$ denotes the projection. The image-range $\left|S_{G}(X)\right|$ of $\bar{\rho}_{n}$ will be denoted by $\left|S_{G}(X)\right|_{n} \subset X_{n+1}$. By the above lemmas, $X_{n}$ and $\rho_{n}$ are neat $G$-CW complexes and $G$-maps, $X_{n}$ is a $G$-CW subcomplex of $X_{n+1}$ and $\rho_{n+1} \mid X_{n}=\rho_{n}$. So, the direct limit

$$
K_{X}=\cup X_{n} \quad \text { and } \quad \rho_{X}=\cup \rho_{n}: K_{X} \rightarrow X
$$

are a neat $G$-CW complex and a $G$-map.

Theorem 1. Let $X$ be a G-space. Then, the $G$-space $K_{X}$ and the $G$-map $\rho_{X}: K_{X} \rightarrow X$ defined above satisfy the following properties.

(1) $K_{X}$ is a neat G-CW complex. Moreover, if $X$ is a $T_{1}$-space, then $K_{X}$ is a Hausdorff G-CW complex.

(2) $\rho_{X}$ is a weak G-homotopy equivalence.

(3) For a G-map $f: X \rightarrow Y$, a canonical G-cellular map $K(f): K_{X} \rightarrow K_{Y}$ is defined and $f \circ \rho_{X}=\rho_{Y} \circ K(f)$. That is, $K$ is a functor from the category of $G$-spaces and G-maps to the category of $G$-CW complexes and G-cellular maps, and $\rho$ is a natural transformation.

(4) If $f$ is an inclusion, then so is $K(f)$.

Proof. (1) $K_{X}$ is already proved to be a neat $G$-CW complex. Suppose that $X$ is a $T_{1}$-space. Then, $X_{1}=\left|S_{G}(X)\right|$ is a Hausdorff $G$-CW complex and we see inductively that so is $X_{n}$. So, the isotropy subgroups at $G$-cells in $K_{X}$ are closed subgroups and hence $K_{X}$ is a Hausdorff $G$-CW complex by Proposition 0.1 .

(2) Let $H$ be a subgroup of $G$. Let $i_{1}: X_{1} \rightarrow K_{X}$ be the natural inclusion. Then, $r_{X}=\rho_{X} \circ i_{1}$. So, by Lemma 1.2 we see that $\left(\rho_{X}\right)_{*}: \pi_{m}\left(K_{X}^{H}, x\right) \rightarrow$ $\pi_{m}\left(X^{H}, \rho_{X}(x)\right)$ is a surjection for every $m \geqq 0$ and any $x \in K_{X}^{H}$.

We shall show that $\left(\rho_{X}\right)_{*}$ is an injection. Let $s:\left(\partial \Delta^{m}, v_{0}\right) \rightarrow\left(K_{X}^{H}, x\right)$ be a map such that $\rho_{X} \circ s$ is based homotopic to null, where $v_{0}=(1,0, \cdots, 0) \in \Delta^{m}$. We may assume that $x \in K_{X}^{0}$. Since $\partial \Delta^{m}$ is compact, $s\left(\partial \Delta^{m}\right) / G$ is contained in $X_{n} / G$ for some $n$. So, $s: \partial \Delta^{m} \rightarrow X_{n}^{H} \subset K_{X}^{H}$ for some $n$. Then, by the $G$-cellular approximation theorem (Proposition 0.2),

$$
\text { Gs: } G / G_{s} \times \partial \Delta^{m} \longrightarrow X_{n}\left(G_{s}=\bigcap_{X \in s\left(\partial \Delta^{m}\right)} G_{X} \supset H\right)
$$

is $G$-homotopic rel. $G / G_{s} \times v_{0}$ to a $G$-cellular map $G s_{1}: G / G_{s} \times \partial \Delta^{m} \rightarrow X_{n}$ and $s_{1}=G s_{1} \mid G_{s} / G_{s} \times \partial \Delta^{m}: \partial \Delta^{m} \rightarrow X_{n}^{G_{s}} \subset X_{n}^{H}$ defines canonically $i_{*}\left(s_{1}\right): \partial \Delta^{m} \rightarrow\left|S_{G}^{c}\left(X_{n}\right)\right|^{H}$ $=\bar{X}_{n}^{H}$ with $\bar{r}_{n} \circ i_{*}\left(s_{1}\right)=s_{1}$ by means of $i_{*}\left(s_{1} \mid \Delta_{i}^{m-1}\right)$ for all faces $\Delta_{i}^{m-1}$ of $\Delta^{m}$. By the assumption on $s, \rho_{n} \circ s_{1}: \partial \Delta^{m} \rightarrow X^{H}$ is based homotopic to null, that is, it is extended to $\bar{s}_{1}: \Delta^{m} \rightarrow X^{H}$. Then, $i_{*}\left(\bar{s}_{1}\right): \Delta^{m} \rightarrow\left|S_{G}(X)\right|^{H}=\left|S_{G}(X)\right|_{n}^{H} \subset X_{n+1}^{H}$ is an extension of $i_{*}\left(\rho_{n} \circ s_{1}\right)=\bar{\rho}_{n} \circ i_{*}\left(s_{1}\right): \partial \Delta^{m} \rightarrow\left|S_{G}(X)\right|_{n}^{H}$. The singular 0-simplex 
$x: \Delta^{0} \rightarrow x \in X_{n}^{H}$ defines $\tilde{x}=i_{*}(x): \Delta^{0} \rightarrow \tilde{x} \in \bar{X}_{n}^{H}$ and $\tilde{x} \times I$ induces an arc $\gamma$ in $X_{n+1}^{H}$ from $x=\bar{r}_{n}(\tilde{x})$ to $\bar{\rho}_{n}(\tilde{x})=\bar{\rho}_{n} \circ i_{*}\left(s_{1}\right)\left(v_{0}\right)$. Let $s_{t}^{\prime}: \partial \Delta^{m} \rightarrow \bar{X}_{n}^{H} \times I, 0 \leqq t \leqq 1$, be the homotopy defined by

$$
s_{t}^{\prime}\left(t_{0}, \cdots, t_{m}\right)= \begin{cases}\left(\tilde{x}, 2-2 t_{0}\right) & \text { if } t \geqq 2-2 t_{0} \\ \left(i_{*}\left(s_{1}\right)\left(2 t_{0} /(2-t), t_{1} \varphi_{t}\left(t_{0}\right), \cdots, t_{m} \varphi_{t}\left(t_{0}\right)\right), t\right) & \text { if } t \leqq 2-2 t_{0}\end{cases}
$$

$\left(\varphi_{t}\left(t_{0}\right)=\left(2-2 t_{0}-t\right) /\left(1-t_{0}\right)(2-t)\right.$ and $\left.\varphi_{0}(1)=1\right)$. Then, the composition $s_{t}^{\prime \prime}: \partial \Delta^{m} \rightarrow$ $\bar{X}_{n}^{H} \times I \rightarrow X_{n+1}^{H}$ of $s_{t}^{\prime}$ and the identification map is a homotopy with $s_{t}^{\prime \prime}\left(v_{0}\right)=$ $x, s_{0}^{\prime \prime}=\bar{r}_{n} \circ i_{*}\left(s_{1}\right)=s_{1}$ and

$$
s_{1}^{\prime \prime}\left(t_{0}, \cdots, t_{m}\right)= \begin{cases}\gamma\left(2-2 t_{0}\right) & \text { if } t_{0} \geqq 1 / 2, \\ \bar{\rho}_{n} \circ i_{*}\left(s_{1}\right)\left(2 t_{0}, t_{1} \varphi_{1}\left(t_{0}\right), \cdots, t_{m} \varphi_{1}\left(t_{0}\right)\right) & \text { if } t_{0} \leqq 1 / 2 .\end{cases}
$$

By the extension $i_{*}\left(\bar{s}_{1}\right): \Delta^{m} \rightarrow X_{n+1}^{H}$ of $\bar{\rho}_{n} \circ i_{*}\left(s_{1}\right)$, the extension of $s_{1}^{\prime \prime}$ over $\Delta^{m}$ is induced. Therefore, $s_{1}$ is based homotopic to null in $X_{n+1}^{H} \subset K_{X}^{H}$ and so is $s$; and we have proved that $\left(\rho_{X}\right)_{*}$ is injective.

(3) For a $G$-map $f: X \rightarrow Y$, $G$-cellular maps $f_{n}: X_{n} \rightarrow Y_{n}$ are defined inductively by $f_{1}=\left|S_{G}(f)\right|$ and $f_{n+1}=\left(f_{n} \amalg \mid S_{G}^{c}\left(f_{n}\right) \times i d \amalg f_{1}\right) / \sim$, which satisfy $\rho_{n+1, Y} \circ f_{n+1}=f \circ \rho_{n, X}$ and $f_{n+1} \mid X_{n}=f_{n}$. So, a $G$-cellular map $K(f)=\cup f_{n}: K_{X}$ $\rightarrow K_{Y}$ is defined and $\rho_{Y} \circ K(f)=f \circ \rho_{X}$.

(4) If $f$ is an inclusion, then so is $f_{1}$. So, we see that $f_{n}$ is an inclusion for any $n$ by induction and so is $K(f)$.

q.e.d.

REMARK. If $f_{0}$ is $G$-homotopic to $f_{1}$, then so is $K\left(f_{0}\right)$ to $K\left(f_{1}\right)$.

Outline of Proof. We use the geometric realization $|S \square(X)|$ of cubic singular complex of $X$ and define $\left|S_{\bar{G}}(X)\right|, K_{\bar{X}}$ and $\rho_{\bar{X}}^{\square}$ in the same way as $\left|S_{G}(X)\right|, K_{X}$ and $\rho_{X}$, respectively. Then, $K_{\bar{X}}^{\square}$ is a $G$-CW complex and $\rho_{X}^{\square}$ is a weak $G$-homotopy equivalence. Moreover, we have a natural inclusion $\iota: K_{\bar{X}}^{\square} \times I \rightarrow K_{\bar{X} \times I}$. Since $\left(\rho_{X}\right)_{*}:\left[K_{\bar{X}}^{\square}, K_{X}\right] \rightarrow\left[K_{\bar{X}}^{\square}, X\right]_{G}$ and $\left(\rho_{\bar{X}}^{\square}\right)_{*}:\left[K_{X}, K_{\bar{X}}^{\square}\right]_{G} \rightarrow$ $\left[K_{X}, X\right]_{G}$ are bijections, we have $G$-maps $\bar{\rho}_{X}: K_{X} \rightarrow K_{\bar{X}}^{\square}$ and $\bar{\rho}_{\bar{X}}^{\square}: K_{\bar{X}} \rightarrow K_{X}$ such that $\rho_{\bar{X}}^{\square} \circ \bar{\rho}_{X} \simeq_{G} \rho_{X}$ and $\rho_{X} \circ \bar{\rho}_{\bar{X}}^{\square} \simeq_{G} \rho_{\bar{X}}^{\square}$. Incidentally this implies $\bar{\rho}_{\bar{X}} \circ \bar{\rho}_{X} \simeq_{G}$ id and $\bar{\rho}_{X} \circ \bar{\rho}_{\bar{X}} \simeq_{G} i d$, because $\left(\rho_{X}\right)_{*}$ and $\left(\rho_{\bar{X}}^{\square}\right)_{*}$ are bijections. Now let $h: X \times I \rightarrow$ $Y$ be a $G$-homotopy connecting $f_{0}$ and $f_{1}$. Then, we get a $G$-homotopy $H$ connecting $K \square\left(f_{0}\right)$ and $K \square\left(f_{1}\right)$ by $H=K \square(h) \circ \iota: K_{\bar{X}}^{\square} \times I \rightarrow K_{\bar{X} \times I}^{\square} \rightarrow K_{\bar{Y}}^{\square}$ such that $\rho_{\bar{Y}}^{\square} \circ H=h \circ\left(\rho_{\bar{Y}}^{\square} \times i d\right)$. We remark that $\bar{\rho}_{\bar{Y}}^{\square} \circ K \square(f) \circ \bar{\rho}_{X}$ is $G$-homotopic to $K(f)$ for any $G$-map $f: X \rightarrow Y$. This is because

$$
\rho_{Y} \circ\left(\bar{\rho}_{\bar{Y}}^{\square} \circ K \square(f) \circ \bar{\rho}_{X}\right) \simeq{ }_{G} \rho_{\bar{Y}}^{\square} \circ K \square(f) \circ \bar{\rho}_{X}=f \circ \rho_{\bar{X}}^{\square} \circ \bar{\rho}_{X} \simeq{ }_{G} f \circ \rho_{X}=\rho_{Y} \circ K(f)
$$

and $\left(\rho_{Y}\right)_{*}:\left[K_{X}, K_{Y}\right]_{G} \rightarrow\left[K_{X}, Y\right]_{G}$ is a bijection. So, $K\left(f_{0}\right)$ is $G$-homotopic to $K\left(f_{1}\right)$. 
The $G$-homotopy type of $K_{X}$ may be found by Brown type construction. In fact, when a $G$-space $X$ is a Hausdorff space and $X^{H}$ is non-empty and arcwise connected for every subgroup $H$ of $G$, a representing Hausdorff $G$-CW complex of the functor $[\cdot, X]_{G, 0}$ is constructed in $[6, \S 6]$ by using Brown's abstruct homotopy theory [1] and has a weak $G$-homotopy type of $X$. Waner [13, p. 357] mentioned that the method can be generalized to the unbased case; two kinds of detailed constructions are given recently in [3] and [8]. In any case Brown type construction does not determine $K_{X}$ uniquely (i.e. is not functorial) and seems inconvenient for proving Theorems 2 and $2^{*}$. Illman also suggested to me to construct $K_{X}$ by gluing singular $G$-simplexes according to his identification; since his identification does not give a $G$-CW complex directly, our construction may be considered as its justification.

\section{$\S 2$. Singular $G$-(co) homology for a weak $G$-homotopy equiva- lence}

We shall show that $\rho_{X}: K_{X} \rightarrow X$ in Theorem 1 induces isomorphisms for the singular $G$-homotopy and $G$-cohomology. First we review the definition of the singular $G$-homology and $G$-homology due to Illman [4].

Definition. A family $\mathscr{F}$ of subgroups of $G$ is called an orbit type family for $G$ if $H \in \mathscr{F}$ implies $g H g^{-1} \in \mathscr{F}$ for any $g \in G$.

Definition. Let $\mathscr{F}$ be an orbit type family for $G$. A covariant (resp. contravariant) $G$-coefficient system for $\mathscr{F}$ is a covariant (resp. contravariant) functor from the category of $G$-spaces $G / H(H \in \mathscr{F})$ and $G$-homotopy classes of $G$-maps to the category of abelian groups.

Let ${ }^{\prime}$ be a covariant $G$-coefficient system for $\mathscr{F}$. For a $G$-space $X$, set

$$
\hat{S}_{G, n}(X ; \mathscr{F})=\left\{T: G / G_{T} \times \Delta^{n} \rightarrow X, G \text {-maps with } G_{T} \in \mathscr{F}\right\} .
$$

Note that $\cup S_{G, n}(X ; \mathscr{F}) \supseteq S_{G}(X)$ even when $\mathscr{F}=\{$ all subgroups of $G\}$. Let $Z(T)$ denote the infinite cyclic group of the generator $T$ and

$$
\hat{C}_{n}^{G}(X ; \mathfrak{f})=\sum_{T \in \hat{S}_{G, n}(X ; \mathscr{F})} Z(T) \otimes \mathfrak{f}\left(G / G_{T}\right) .
$$

Let $T^{(i)}=T \circ\left(i d \times \delta_{i}\right)$ and define $\hat{\partial}: C_{n}^{G}(X ; \mathfrak{i}) \rightarrow \hat{G}_{n-1}^{G}(X ; \mathfrak{l})$ by the linear extension of $\hat{\partial}(T \otimes a)=\sum(-1)^{i} T^{(i)} \otimes a$. For $T, T^{\prime} \in \hat{S}_{G, n}(X, \mathscr{F})$ we now define that

$$
T \sim T^{\prime} \text { by } h: G / G_{T} \times \Delta^{n} \longrightarrow G / G_{T^{\prime}} \times \Delta^{n}
$$

if $h$ is a $G$-map covering $i d: \Delta^{n} \rightarrow \Delta^{n}$ (i.e. $p r \circ h=p r$ ) and $T=T^{\prime} \circ h$. Let 
$\bar{C}_{n}^{G}(X ; \mathfrak{f})$ be the submodule of $\hat{C}_{n}^{G}(X ; \mathfrak{f})$ generated by the elements

$$
T \otimes a-T^{\prime} \otimes a^{\prime} \quad \text { with } T \sim T^{\prime} \text { by } h \text { and } h_{*}(a)=a^{\prime} .
$$

We define the chain complex $\left(C_{*}^{G}(X ; \mathfrak{f}), \partial\right)$ by $C_{n}^{G}(X ; \mathfrak{f})=\hat{C}_{n}^{G}(X ; \mathfrak{f}) / \bar{C}_{n}^{G}(X ; \mathfrak{f})$ and $\partial=$ the homomorphism induced by $\hat{\partial}$. Then, the singular $G$-homology $H_{n}^{G}(X ; \mathfrak{l})$ is the $n$-th homology module of the chain complex $\left(C_{*}^{G}(X ; \mathfrak{l}), \partial\right)$. Moreover, if $A$ is a $G$-invariant subspace of $X$, then we define

$$
C_{n}^{G}(X, A ; \mathfrak{l})=C_{n}^{G}(X ; \mathfrak{f}) / C_{n}^{G}(A ; \mathfrak{l}) \quad \text { and } \quad H_{n}^{G}(X, A ; \mathfrak{l})=H_{n}\left(C_{*}^{G}(X, A ; \mathfrak{f}), \partial\right) \text {. }
$$

Let $\mathrm{m}$ be a contravariant $G$-coefficient system for $\mathscr{F}$. To define the singular $G$-cohomology, we introduce a covariant $G$-coefficient system $Z_{\mathscr{F}}$ defined by $Z_{\mathscr{F}}(G / H)=Z$ for $H \in \mathscr{F}$, i.e., $\hat{C}_{n}^{G}\left(X ; Z_{\mathscr{F}}\right)=\sum Z(T)$. Let $\hat{C}_{G}^{n}(X ; \mathfrak{m})=$ $\left\{c^{*}: \hat{C}_{n}^{G}\left(X ; Z_{\mathscr{F}}\right) \rightarrow \sum \mathfrak{m}(G / H)\right.$, homomorphisms with $\left.c^{*}(T) \in \mathfrak{m}\left(G / G_{T}\right)\right\}$ and $\hat{\delta}: \hat{C}_{G}^{n-1}(X ; \mathfrak{m}) \rightarrow \hat{C}_{G}^{n}(X ; \mathfrak{m})$ be defined by $\hat{\delta}\left(c^{*}\right)(T)=c^{*}(\hat{\partial} T)$. Let $C_{G}^{n}(X ; \mathfrak{m})$ be the submodule of $\hat{C}_{G}^{n}(X ; \mathfrak{m})$ consisting of all the elements $c^{*}$ which satisfy the following condition: If $T \sim T^{\prime}$ by $h$, then $c^{*}(T)=h^{*} c^{*}\left(T^{\prime}\right)$, where $h^{*}: \mathfrak{m}\left(G / G_{T^{\prime}}\right) \rightarrow \mathfrak{m}\left(G / G_{T}\right)$ is the homomorphism induced by $h$. The singular $G$-cohomology $H_{G}^{n}(X ; \mathfrak{m})$ is then the $n$-th cohomology module of the cochain complex $\left(C_{G}^{*}(X ; \mathfrak{m}), \delta\right)$ where $\delta=\hat{\delta} \mid C_{G}^{n}(X ; \mathfrak{m})$. Moreover, if $A$ is a $G$-invariant subspace of $X$, then $C_{G}^{n}(X, A ; \mathfrak{m})=\operatorname{Ker}\left(C_{G}^{n}(X ; \mathfrak{m}) \rightarrow C_{G}^{n}(A ; \mathfrak{m})\right)$ and $H_{G}^{n}(X, A ; \mathfrak{m})$ $=H^{n}\left(C_{G}^{*}(X, A ; \mathfrak{m}), \delta\right)$.

In [4], the basic properties of the singular $G$-(co)homology are proved which will be used in this paper.

Now we present the precise form of Theorems 2 and $2^{*}$. Let $X$ be a $G$ space and $A$ a $G$-invariant subspace of $X$. Then, by Theorem 1 , we have the pair $\left(K_{X}, K_{A}\right)$ of $G$-CW complex $K_{X}$ and its $G$-CW subcomplex $K_{A}$ and the pair of the $G$-maps

$$
\rho=\left(\rho_{X}, \rho_{A}\right):\left(K_{X}, K_{A}\right) \longrightarrow(X, A) \text { with } \rho_{X} \mid K_{A}=\rho_{A} .
$$

Let $\mathfrak{i}$ (resp. $\mathfrak{m})$ be a covariant (resp. contravariant) $G$-coefficient system for an orbit type family $\mathscr{F}$ for $G$.

Theorem 2. The induced homomorphism $\rho_{*}: H_{n}^{G}\left(K_{X}, K_{A} ; \mathfrak{l}\right) \rightarrow H_{n}^{G}(X, A ; \mathfrak{l})$ on the singular $G$-homology is an isomorphism for every $n$.

THEOREM $2^{*}$. The induced homomorphism

$$
\rho^{*}: H_{G}^{n}(X, A ; \mathfrak{m}) \longrightarrow H_{G}^{n}\left(K_{X}, K_{A} ; \mathfrak{m}\right)
$$

on the singular G-cohomology is an isomorphism for every $n$.

By Theorem 1 and the homotopy axiom of the singular $G$-(co)homology, 
Theorems 2 and $2^{*}$ can be reformulated in a simpler form.

Corollary. Let $h:(X, A) \rightarrow(Y, B)$ be a weak G-homotopy equivalence between pairs of $G$-spaces. Then, the induced homomorphisms $h_{*}: H_{n}^{G}(X, A ; \mathfrak{l})$ $\rightarrow H_{n}^{G}(Y, B ; \mathfrak{l})$ and $h^{*}: H_{G}^{n}(Y, B ; \mathfrak{m}) \rightarrow H_{G}^{n}(X, A ; \mathfrak{m})$ on the singular $G$-homology and $G$-cohomology are isomorphisms for every $n$.

Proof of Corollary. Since $K(h):\left(K_{X}, K_{A}\right) \rightarrow\left(K_{Y}, K_{B}\right)$ is a weak $G$ homotopy equivalence between the pairs of $G$-CW complexes by Theorem 1 and the assumption, $K(h)$ is a $G$-homotopy equivalence by the theorem of J.H.C. Whitehead (Proposition 0.2). So,

$$
K(h)_{*}: H_{n}^{G}\left(K_{X}, K_{A} ; \mathfrak{f}\right) \longrightarrow H_{n}^{G}\left(K_{Y}, K_{B} ; \mathfrak{l}\right)
$$

and

$$
K(h)^{*}: H_{G}^{n}\left(K_{Y}, K_{B} ; \mathfrak{m}\right) \longrightarrow H_{G}^{n}\left(K_{X}, K_{A} ; \mathfrak{m}\right)
$$

are isomorphisms by the homotopy axiom. Hence, Corollary follows from Theorems 2 and $2^{*}$. q.e.d.

In the rest of this section, we shall prove Theorems 2 and $2^{*}$ in the case when $A=\phi$. Then, we have the theorems because of the long exact sequences of the (co)homology modules and the 5-lemma.

By using $X_{m}(1 \leqq m<\infty)$ in the definition of $K_{X}=\cup X_{m}$, we abbreviate as follows: $\hat{C}=\hat{C}_{0} \supset \bar{C}=\bar{C}_{0}, \hat{C}_{m}=\sum_{n \geqq 0} \hat{C}_{n}^{G}\left(X_{m} ; \mathfrak{l}\right) \supset \bar{C}_{m}=\sum_{n \geqq 0} \bar{C}_{n}^{G}\left(X_{m} ; \mathfrak{l}\right)$ for $0 \leqq m$ $\leqq \infty$, where $X_{0}=X$ and $X_{\infty}=K_{X}$. Let $T: G / G_{T} \times \Delta^{n} \rightarrow X$ be a $G$-map with $G_{T} \in \mathscr{F}$. Define $\sigma=T \mid G_{T} / G_{T} \times \Delta^{n} \rightarrow X$ and we get a singular $G$-simplex $G \sigma: G / G_{\sigma} \times \Delta^{n} \rightarrow X$ contained in $S_{G}(X)$. Note that $G_{T} \subset G_{\sigma}$. Then, by composing the projection $G / G_{T} \rightarrow G / G_{\sigma}$ and the canonical $G$-map $i_{*}(G \sigma): G / G_{\sigma} \times$ $\Delta^{n} \rightarrow\left|S_{G}(X)\right|$, we have a $G$-map

$$
i_{*}(T): G / G_{T} \times \Delta^{n} \rightarrow\left|S_{G}(X)\right|=X_{1} \subset X_{m} \subset X_{\infty}=K_{X} \quad(1 \leqq m \leqq \infty)
$$

with $\rho_{m} \circ i_{*}(T)=T$ where $\rho_{1}=r_{X}$ and $\rho_{\infty}=\rho_{X}$. So, we have the homomorphisms.

$$
i_{*}: \hat{C} \rightarrow \hat{C}_{1} \rightarrow \hat{C}_{m}(1 \leqq m \leqq \infty) \text { given by } i_{*}(T \otimes a)=i_{*}(T) \otimes a
$$

which is a chain map and satisfies $\rho_{*} \circ i_{*}=i d$ for the chain map

$$
\rho_{*}: \hat{C}_{m} \rightarrow \hat{C}(1 \leqq m \leqq \infty) \text { given by } \rho_{*}(S \otimes a)=\rho_{*}(S) \otimes a \text {. }
$$

Clearly $\rho_{*}\left(\bar{C}_{m}\right) \subset \bar{C}$. To study $i_{*}(\bar{C})$, we prepare some lemmas. Let

$$
i_{m-1}: X_{m-1} \rightarrow \bar{X}_{m-1}=\left|S_{G}^{c}\left(X_{m-1}\right)\right| \text { and } j_{m}: \bar{X}_{m-1} \times I \rightarrow X_{m} \quad(2 \leqq m \leqq \infty)
$$

be the canonical inclusion $i_{m-1}=i_{*}$ given in Lemma 1.3 and the identification 
map to the double mapping cylinder $X_{m}$, respectively. For any $G$-map $S: G / G_{S} \times \Delta^{n} \rightarrow X_{m-1}$ with $G_{S} \in \mathscr{F}$, we define a $G$-map

$$
(S \times I)_{m}=j_{m} \circ\left(\left(i_{m-1} \circ S\right) \times i d_{I}\right): G / G_{S} \times \Delta^{n} \times I \rightarrow X_{m-1} \times I \rightarrow \bar{X}_{m-1} \times I \rightarrow X_{m} .
$$

Subdivide the product $\Delta^{n} \times I$ into $(n+1)$-simplexes $\Delta^{n+1}$ s by ordering the vertexes and we get a chain

$$
\langle S \times I\rangle_{m} \in \hat{C}_{n+1}^{G}\left(X_{m} ; Z_{\mathscr{F}}\right) \subset \hat{C}_{n+1}^{G}\left(K_{X} ; Z_{\mathscr{F}}\right)
$$

as the sum of $G$-maps $(S \times I)_{m} \mid G / G_{S} \times \Delta^{n+1}$ 's. So, for any chain $d=\sum S_{i} \otimes a_{i}$ $\in \hat{C}_{n}^{G}\left(X_{m-1}, k\right) \subset \hat{C}_{m-1}(m \geqq 2)$, we can define

$$
\langle d \times I\rangle_{m}=\sum\left\langle S_{i} \times I\right\rangle_{m} \otimes a_{i} \in \hat{C}_{n+1}^{G}\left(X_{m} ; k\right) \subset \hat{C}_{m} \subset \hat{C}_{\infty}
$$

Let $T: G / G_{T} \times \Delta^{n} \rightarrow X$ be a $G$-map with $G_{T} \in \mathscr{F}$. Then, by using $\left|S_{G}(X)\right|=$ $\left|S_{G}(X)\right|_{m-1} \subset X_{m}$ (see the definition of $K_{X}$ ) instead of $\left|S_{G}(X)\right|=X_{1} \subset X_{m}$ in the definition of $i_{*}(T)$, we have

$$
i_{*}^{m}(T) ; G / G_{T} \times \Delta^{n} \rightarrow\left|S_{G}(X)\right|=\left|S_{G}(X)\right|_{m-1} \subset X_{m} \quad \text { for any } 2 \leqq m<\infty
$$

with $\rho_{m} \circ \dot{i}_{*}^{m}(T)=T$. So, we also have a chain map

$$
i_{*}^{m}: \hat{C} \rightarrow \hat{C}_{m} \quad \text { with } \rho_{*} \circ i_{*}^{m}=i d \text { given by } i_{*}^{m}(T \otimes a)=i_{*}^{m}(T) \otimes a .
$$

Lemma 2.1. For any $d \in \hat{C}_{m-1}(m \geqq 2)$,

$$
\hat{\partial}\langle d \times I\rangle_{m}=d-i_{*}^{m} \rho_{*} d-\langle\hat{\partial} d \times I\rangle_{m} \quad \text { in } \hat{C}_{m} \subset \hat{C}_{\infty} \text {. }
$$

Proof. By the definition of $(S \times I)_{m},(S \times I)_{m} \mid G / G_{S} \times \Delta^{n} \times t$ is $\bar{r}_{m-1} \circ i_{m-1}$ $\circ S=S$ for $t=0$ and $\bar{\rho}_{m-1} \circ i_{m-1} \circ S=\left|S_{G}\left(\rho_{m-1}\right)\right| \circ i_{*}(S)=i_{*}^{m}\left(\rho_{m-1} \circ S\right)$ for $t=1$. So, we get the lemma. q.e.d.

Now let $T: G / G_{T} \times \Delta^{n} \rightarrow X$ and $T^{\prime}: G / G_{T^{\prime}} \times \Delta^{n} \rightarrow X$ be $G$-maps $\left(G_{T}, G_{T^{\prime}} \in \mathscr{F}\right)$ such that $T\left(=T^{\prime} \circ h\right) \sim T^{\prime}$ by $h: G / G_{T} \times \Delta^{n} \rightarrow G / G_{T^{\prime}} \times \Delta^{n}$ covering id; $\Delta^{n} \rightarrow \Delta^{n}$. Then, for any $2 \leqq m<\infty$, we have $G$-maps $i_{*}(T), i_{*}\left(T^{\prime}\right) \circ h: G / G_{T} \times \Delta^{n} \rightarrow\left|S_{G}(X)\right|$ $=X_{1} \subset X_{m-1}$ and chains

$$
\begin{aligned}
& e\left(T, T^{\prime}\right)_{m}=\left\langle i_{*}(T) \times I\right\rangle_{m}-\left\langle\left(i_{*}\left(T^{\prime}\right) \circ h\right) \times I\right\rangle_{m}, \\
& e\left(\hat{\partial} T, \hat{\partial} T^{\prime}\right)_{m}=\left\langle i_{*} \hat{\partial} T \times I\right\rangle_{m}-\left\langle\left(i_{*} \hat{\partial} T^{\prime} \circ h\right) \times I\right\rangle_{m}
\end{aligned}
$$

in $\hat{C}_{\ell}^{G}\left(X_{m} ; Z_{\mathscr{F}}\right) \subset \hat{C}_{\ell}^{G}\left(K_{X} ; Z_{\mathscr{F}}\right)$ for $\ell=n+1, n$, respectively, where $i_{*} \hat{\partial} T^{\prime} \circ h$ means $\sum(-1)^{j} i_{*}\left(T^{\prime(j)}\right) \circ h^{(j)}\left(h^{(j)}\right.$ is the restriction of $\left.h\right)$.

LEMMA 2.2. In $\hat{C}_{m} \subset \hat{C}_{\infty}$ and $\hat{C}$ with $\mathfrak{f}=Z_{\mathscr{F}}$,

$$
\hat{\partial} e\left(T, T^{\prime}\right)_{m}=i_{*}(T)-i_{*}\left(T^{\prime}\right) \circ h-e\left(\hat{\partial} T, \hat{\partial} T^{\prime}\right)_{m} \quad \text { and } \quad \rho_{*} e\left(T, T^{\prime}\right)_{m}=0
$$

for any $2 \leqq m<\infty$. 
Proof. We see the first equality by Lemma 2.1 for $d=i_{*}(T)$ and $i_{*}\left(T^{\prime}\right) \circ h$. The second one follows, because $\rho_{m} \circ\left(i_{*}(T) \times I\right)_{m}$ and $\rho_{m} \circ\left(\left(i_{*}\left(T^{\prime}\right) \circ h\right)\right.$ $\times I)_{m}$ are both $r_{X} \circ i_{*}(T)=T=T^{\prime} \circ h=r_{X} \circ\left(i_{*}\left(T^{\prime}\right) \circ h\right)$ on $G / G_{T} \times \Delta^{n} \times t$ for any $t \in I$.

q.e.d.

Lemma 2.3. For any $c \in \bar{C}$ and $2 \leqq m<\infty$, there exists $e_{m} \in \hat{C}_{m} \subset \hat{C}_{\infty}$ such that

$$
i_{*} c-\left\langle i_{*} \hat{\partial} c \times I\right\rangle_{m}-\hat{\partial} e_{m} \in \bar{C}_{m} \subset \bar{C}_{\infty} \quad \text { and } \quad \rho_{*} e_{m}=0 .
$$

Proof. Put $c=\sum\left(T_{i} \otimes a_{i}-T_{i}^{\prime} \otimes a_{i}^{\prime}\right)$ where $T_{i} \sim T_{i}^{\prime}$ by $h_{i}$ and $h_{i *}\left(a_{i}\right)=a_{i}^{\prime}$. Then, $i_{*} c=\sum\left(i_{*}\left(T_{i}\right) \otimes a_{i}-i_{*}\left(T_{i}^{\prime}\right) \otimes a_{i}^{\prime}\right)$ is equal to

$$
\begin{gathered}
\sum \hat{\partial} e\left(T_{i}, T_{i}^{\prime}\right) \otimes a_{i}+\sum\left\{\left(i_{*}\left(T_{i}^{\prime}\right) \circ h_{i}\right) \otimes a_{i}-i_{*}\left(T_{i}^{\prime}\right) \otimes a_{i}^{\prime}\right\}+\left\langle i_{*} \hat{\partial} c \times I\right\rangle_{m} \\
-\sum\left\{\left\langle\left(i_{*} \hat{\partial} T_{i}^{\prime} \circ h_{i}\right) \times I\right\rangle_{m} \otimes a_{i}-\left\langle i_{*} \hat{\partial} T_{i}^{\prime} \times I\right\rangle_{m} \otimes a_{i}^{\prime}\right\}
\end{gathered}
$$

by the first equality in Lemma 2.2, because the sum of the last two terms is $\sum e\left(\hat{\partial} T_{i}, \hat{\partial} T_{i}^{\prime}\right)_{m} \otimes a_{i}$. The second and the last terms are in $\bar{C}_{1} \subset \bar{C}_{m}$ and $\bar{C}_{m}$, respectively. So, we have the lemma by taking $e_{m}=\sum e\left(T_{i}, T_{i}^{\prime}\right)_{m} \otimes a_{i}$. q.e.d.

Proof of Theorem 2. Surjectivity of $\rho_{*}: H_{*}\left(\hat{C}_{\infty} / \bar{C}_{\infty}\right) \rightarrow H_{*}(\hat{C} / \bar{C})$ : Suppose that $c \in \hat{C}$ represents a cycle in $\hat{C} / \bar{C}$, i.e., $\hat{\partial} c \in \bar{C}$. Then, $\hat{\partial}\left(i_{*} c-e\right) \in \bar{C}_{\infty}$ with $\rho_{*} e=0$ by Lemma 2.3. So, $\rho_{*}\left(i_{*} c-e\right)=c$; and $\rho_{*}$ is surjective.

Injectivity of $\rho_{*}$ : Suppose that $d \in \hat{C}_{\infty}$ satisfies $\hat{\partial} d \in \bar{C}_{\infty}$ and $\rho_{*} d \in \hat{\partial} \hat{C}+\bar{C}$. Then, we can choose $m \geqq 2$ so that $d \in \hat{C}_{m-1}$ and $\hat{\partial} d \in \bar{C}_{m-1}$, and $c \in \hat{C}$ and $\bar{c} \in \bar{C}$ so that $\rho_{*} d=\hat{\partial} c+\bar{c}$. Put $d^{\prime}=d-i_{*} \rho_{*} d=d-i_{*} \hat{\partial} c-i_{*} \bar{c} \in \hat{C}_{m-1}$. Then, $\rho_{*} d^{\prime}=0$, $\hat{\partial} d^{\prime}=\hat{\partial} d-i_{*} \hat{\partial} \bar{c}$ and

$$
\hat{\partial}\left\langle d^{\prime} \times I\right\rangle_{m}=d^{\prime}-\left\langle\hat{\partial} d^{\prime} \times I\right\rangle_{m}=d-\hat{\partial} i_{*} c-\langle\hat{\partial} d \times I\rangle_{m}-\left(i_{*} \bar{c}-\left\langle i_{*} \hat{\partial} \bar{c} \times I\right\rangle_{m}\right)
$$

by Lemma 2.1. Here, $\langle\hat{\partial} d \times I\rangle_{m} \in \bar{C}_{m} \subset \bar{C}_{\infty}$ since $\hat{\partial} d \in \bar{C}_{m-1}$, and $i_{*} \bar{c}-\left\langle i_{*} \hat{\partial} \bar{c} \times I\right\rangle_{m}$ $\in \hat{\partial} \hat{C}_{\infty}+\bar{C}_{\infty}$ by Lemma 2.3. So, $d \in \hat{\partial} \hat{C}_{\infty}+\bar{C}_{\infty}$; and $\rho_{*}$ is injective. q.e.d.

To prove Theorem $2^{*}$, we use the following abbreviation: $\hat{C}^{*}=\hat{C}_{0}^{*}$, $C^{*}=C_{0}^{*}, \hat{C}_{m}^{*}=\sum_{n \geqq 0} \hat{C}_{G}^{n}\left(X_{m} ; \mathfrak{m}\right), C_{X}^{*}=\sum_{n \geqq 0} C_{G}^{n}\left(X_{m} ; \mathfrak{m}\right)(0 \leqq m \leqq \infty)$, where $X_{0}=$ $X$ and $X_{\infty}=K_{X}$. There are the cochian maps

$$
i^{*}: \hat{C}_{m}^{* \rightarrow} \rightarrow \hat{C}, \rho_{m}^{*} \rightarrow \hat{C}^{*} \rightarrow \hat{C}_{m}^{*} \quad \text { and } \quad \iota_{m, \ell}^{*}: \hat{C}_{\ell}^{*} \rightarrow \hat{C}_{m}^{*} \quad(1 \leqq m \leqq \ell \leqq \infty)
$$

given by $i^{*} d^{*}(T)=d^{*}\left(i_{*}(T)\right), \rho_{m}^{*} c^{*}(S)=c^{*}\left(\rho_{m} \circ S\right)$ and $\iota_{m, \ell}^{*} d^{*}(S)=d^{*}(S)$. Clearly $i^{*} \circ \rho_{m}^{*}=i d, i^{*} \circ \iota_{m, \ell}^{*}=i^{*}, \iota_{m, \ell}^{*} \circ \rho_{\ell}^{*}=\rho_{m}^{*}$ and $\rho_{m}^{*}\left(C^{*}\right) \subset C_{m}^{*}, \iota_{m, \ell}^{*}\left(C_{\ell}^{*}\right)=C_{m}^{*}$. For any $1 \leqq m<\ell \leqq \infty$ and $d^{*} \in C_{\ell}^{*}$, we define $\left\langle d^{*} \times I\right\rangle_{m}^{*}=\left\langle\iota_{m+1, \ell}^{*} d^{*} \times I\right\rangle_{m}^{*} \in C_{m}^{*}$ by

$$
\left\langle d^{*} \times I\right\rangle_{m}^{*}(S)=d^{*}\left(\langle S \times I\rangle_{m+1}\right) .
$$


LEMmA 2.4. Let $1 \leqq m<\ell \leqq \infty$ and $d^{*} \in \hat{C}_{\ell}^{*}$.

(1) If $d^{*} \in C_{\ell}^{*}$, then $\left\langle d^{*} \times I\right\rangle_{m}^{*} \in C_{m}^{*}$.

(2) If $\delta d^{*} \in \rho_{\ell}^{*} \hat{C}^{*}$ in addition, then $e^{*}\left(d^{*}, m\right)$, defined by $i^{*} d^{*}-$ $\hat{\delta} i^{*}\left\langle d^{*} \times I\right\rangle_{m}^{*}$, is contained in $C^{*}$.

(3) If $\delta d^{*}=0$ in addition, then $\delta e^{*}\left(d^{*}, m\right)=0$ and

$$
\rho_{m}^{*} e^{*}\left(d^{*}, m\right)=\iota_{m, \ell}^{*} d^{*}-\delta\left\langle d^{*} \times I\right\rangle_{m}^{*} \quad \text { in } \quad C_{m}^{*} .
$$

Proof. (1) is clear. (2) Let $T \sim T^{\prime}$ by $h$. Then,

$$
e^{*}\left(d^{*}, m\right)(T)=d^{*}\left(i_{*}(T)-\left\langle i_{*} \hat{\partial} T \times I\right\rangle_{m+1}\right)
$$

is equal to

$$
\delta d^{*}\left(e\left(T, T^{\prime}\right)_{m+1}\right)+d^{*}\left(i_{*}\left(T^{\prime}\right) \circ h-\left\langle\left(i_{*} \hat{\partial} T^{\prime} \circ h\right) \times I\right\rangle_{m+1}\right)
$$

and to $h^{*}\left(e^{*}\left(d^{*}, m\right)\left(T^{\prime}\right)\right)$ by Lemma 2.2 and the assumption that $d^{*} \in C_{\ell}^{*}$ and $\delta d^{*} \in \rho_{\ell}^{*} \hat{C}^{*}$. So, (2) holds. (3) The first equality is clear. Let $d \in \hat{C}_{m}$ for $\mathfrak{I}=Z_{\mathscr{F}}$. Then,

$$
\begin{aligned}
& \left(\iota_{m, \ell}^{*} d^{*}-\rho_{m}^{*} e^{*}\left(d^{*}, m\right)\right)(d)=d^{*}\left(d-i_{*} \rho_{*} d+\left\langle i_{*} \rho_{*} \hat{\partial} d \times I\right\rangle_{m+1}\right) \\
& \quad=d^{*}\left(\hat{\partial}\left\langle\left(d-i_{*} \rho_{*} d\right) \times I\right\rangle_{m+1}+\langle\hat{\partial} d \times I\rangle_{m+1}\right)=\delta\left\langle d^{*} \times I\right\rangle_{m}^{*}(d)
\end{aligned}
$$

by Lemma 2.1 and $\delta d^{*}=0$. So, we see the second equality.

q.e.d.

LEMma 2.5. The induced homomorphisms $\left(\iota_{m, \infty}^{*}\right)^{*}: H^{*}\left(C_{\infty}^{*}\right) \rightarrow H^{*}\left(C_{m}^{*}\right)(1 \leqq$ $m<\infty)$ induce the isomorphism $\lim \left(\iota_{\infty}^{*}\right)^{*}$ of $H^{*}\left(C_{\infty}^{*}\right)$ onto the inverse limit of $\left\{H^{*}\left(C_{m}^{*}\right) ;\left(\iota_{m, \ell}^{*}\right)^{*}: H^{*}\left(C_{\ell}^{*}\right) \rightarrow H^{*}\left(C_{m}^{*}\right)(1 \leqq m \leqq \ell<\infty)\right\}$.

Proof. Suppose that $d^{*} \in C_{m}^{*}$ with $\delta d^{*}=0$ represents $\left[d^{*}\right] \in \operatorname{Im}\left(\iota_{m, m+1}^{*}\right)^{*}$. Then, we have $d^{\prime *} \in C_{m+1}^{*}$ with $\delta d^{* *}=0$ and $\left[\iota_{m, m+1}^{*} d^{*}\right]=\left[d^{*}\right]$ in $H^{*}\left(C_{m}^{*}\right)$. So, Lemma 2.4 (3) shows that $e^{*}=e^{*}\left(d^{\prime *}, m\right) \in C^{*}$ is a cocycle and, for any $\ell$ with $m<\ell<\infty$,

$$
\left[d^{*}\right]=\left[\iota_{m, m+1}^{*} d^{* *}\right]=\left[\rho_{m}^{*} e^{*}\right]=\left(\iota_{m, \ell}^{*}\right)^{*}\left[\rho_{\ell}^{*} e^{*}\right] \in \operatorname{Im}\left(\iota_{m, \ell}^{*}\right)^{*} .
$$

Hence, the inverse system $\left\{H^{*}\left(C_{m}^{*}\right)\right\}$ satisfies the Mittag-Leffler condition (ML). Thus, $\lim { }^{1} H^{*}\left(C_{m}^{*}\right)=0$ as is well-known (e.g., [12, p. 131]). So, the lemma follows from the following exact sequence:

$$
0 \rightarrow \lim { }^{1} H^{*-1}\left(C_{m}^{*}\right) \rightarrow H^{*}\left(C_{\infty}^{*}\right) \rightarrow \lim H^{*}\left(C_{m}^{*}\right) \rightarrow 0 .
$$

In fact, since $H^{*}\left(C_{m}^{*}\right)=H_{G}^{*}\left(X_{m}\right)$ and $X_{\infty}=K_{X}$ has the $G$-homotopy type of a G-CW complex

$$
X_{1} \times[0,1] \cup X_{2} \times[1,2] \cup \cdots \cup X_{n} \times[n-1, n] \cup \cdots
$$


contained in $X_{\infty} \times[0, \infty]$, the proof of Lemma 2 in [9] can be applied to demonstrate the exact sequence by using the additivity property and MayerVietoris sequences for $H_{G}^{*}$.

q.e.d.

Proof of Theorem $2^{*}$. Injectivity of $\rho^{*}=\left(\rho_{\ell}^{*}\right)^{*}: H^{*}\left(C^{*}\right) \rightarrow H^{*}\left(C_{\ell}^{*}\right)(2 \leqq \ell$ $\leqq \infty)$ : Suppose that $c^{*} \in C^{*}$ with $\delta c^{*}=0$ satisfies $\left[\rho_{l}^{*} c^{*}\right]=0$ in $H^{*}\left(C^{*}\right)$. Then, $\rho_{\ell} c^{*}=\delta d^{*}$ for some $d^{*} \in C_{\ell}^{*}$ and $e^{*}\left(d^{*}, m\right) \in C^{*}(1 \leqq m<\ell)$ in Lemma 2.4 (2) satisfies $\delta e^{*}\left(d^{*}, m\right)=\hat{\delta} i^{*} d^{*}=i^{*} \rho_{\ell}^{*} c^{*}=c^{*}$. So, $\left[c^{*}\right]=0$ in $H^{*}\left(C^{*}\right)$; and $\rho^{*}=$ $\left(\rho_{\ell}^{*}\right)^{*}$ is injective.

Surjectivity of $\rho^{*}=\left(\rho_{\infty}^{*}\right)^{*}$ : Let $d^{*} \in C_{\infty}^{*}$ be a cocycle. Then, for any $2 \leqq$ $\ell \leqq m<\infty$, Lemma 2.4 (3) shows that $e_{m}^{*}=e^{*}\left(d^{*}, m\right) \in C^{*}$ is a cocycle and

$$
\left(\rho_{\ell}^{*}\right)^{*}\left[e_{m}^{*}\right]=\left(\iota_{\ell, m}^{*}\right) *\left[\rho_{m}^{*} e_{m}^{*}\right]=\left(\iota_{\ell, m}^{*}\right) *\left[\iota_{m, \infty}^{*} d^{*}\right]=\left(\iota_{\ell, \infty}^{*}\right) *\left[d^{*}\right]
$$

in $H^{*}\left(C_{\ell}^{*}\right)$. Since $\left(\rho_{\ell}^{*}\right)^{*}$ is injective as is proved already, this shows that $\left[e_{m}^{*}\right]=\left[e_{2}^{*}\right]$ in $H^{*}\left(C^{*}\right)$ and

$$
\left(\iota_{m, \infty}^{*}\right) *\left[\rho_{\infty}^{*} e_{2}^{*}\right]=\left(\rho_{m}^{*}\right)^{*}\left[e_{m}^{*}\right]=\left(\iota_{m, \infty}^{*}\right) *\left[d^{*}\right] \quad \text { in } H^{*}\left(C_{m}^{*}\right)
$$

for any $2 \leqq m<\infty$. Therefore, $\left[d^{*}\right]=\left(\rho_{\infty}^{*}\right)^{*}\left[e_{2}^{*}\right]$ in $H^{*}\left(C_{\infty}^{*}\right)$ by Lemma 2.5 ; and $\rho^{*}=\left(\rho_{\infty}^{*}\right)^{*}$ is surjective.

q.e.d.

Thus, the proofs of Theorems 2 and $2^{*}$ are completed.

\section{§ 3. Singular $G$-(co) homology via $K_{X}$}

A $G$-coefficient system for an orbit type family $\mathscr{F}$ is called generic with respect to a $G$-space $X$, if $G_{x} \in \mathscr{F}$ for every $x \in X$. When $(X, A)$ is a pair of $G$-CW complexes and the $G$-coefficient system is generic with respect to $X$, the cellular $G$-(co) homology of $(X, A)$ can be defined as is done in [7]; moreover, it is mentioned there that any classical $G$-cohomology theory on $(X, A)$ with additivity axiom coincides with the cellular $G$-cohomology theory.

So, in case the $G$-coefficient system is generic with respect to $K_{X}$, for example, if $\mathscr{F}=\{$ all subgroups of $G\}$, we can define another $G$-(co) homology theory of singular type on a pair $(X, A)$ of $G$-spaces by the cellular $G$-(co) homology of $\left(K_{X}, K_{A}\right)$ without any aid from the singular $G$-(co) homology of Illman [4]; and these two G-(co) homologies of singular type coincide with each other. The aim of this section is to give a detailed proof of this fact.

THEOREM 3. Let $(X, A)$ be a pair of $G$-spaces. Assume that the G-coefficient system for $\mathscr{F}$ is generic with respect to $K_{X}$. Then, the singular $G$ homology and G-cohomology of $(X, A)$ in the sense of Illman [4] coincide with the cellular G-homology and $G$-cohomology of $\left(K_{X}, K_{A}\right)$, respectively.

REMARK. The assumption of Theorem 3 is satisfied, either if $\mathscr{F}=\{$ all 
subgroups of $G\}$, or if $X$ is a $T_{1}$-space and $\mathscr{F}=\{$ all closed subgroups of $G\}$ (cf. Theorem 1).

By Theorems 2 and $2^{*}$ and the dimension axiom on the singular $G$-(co) homology [4], it suffices to show the following proposition.

Proposition 3.1. Let $(X, A)$ be a pair of $G$-CW complexes. Let $¥$ (resp. $\mathrm{m})$ be a covariant (resp. contravariant) G-coefficient system for an orbit type family $\mathscr{F}$. Then, there are strongly convergent spectral sequences,

$$
H_{p}^{G, c}\left(X, A ; \mathfrak{f}_{q}\right) \Rightarrow H_{p+q}^{G}(X, A ; \mathfrak{\mathfrak { l }}) \quad \text { and } \quad H_{G}^{p, c}\left(X, A ; \mathfrak{m}^{q}\right) \Rightarrow H_{G}^{p+q}(X, A ; \mathfrak{m})
$$

where $\mathfrak{\mathfrak { F }}_{q}$ and $\mathfrak{m}^{q}$ are the G-coefficient systems for the orbit type family consisting of all subgroups of $G$ defined by $\mathfrak{f}_{q}(G / H)=H_{q}^{q}(G / H ; \mathfrak{i})$ and $\mathfrak{m}^{q}(G / H)=$ $H_{G}^{q}(G / H ; \mathfrak{m})$, and $H_{p}^{G, c}\left(X, A ; \mathfrak{x}_{q}\right)\left(\right.$ resp. $\left.H_{G}^{p, c}\left(X, A ; \mathfrak{m}^{q}\right)\right)$ denotes the cellular $G$ homology (resp. G-cohomology) of $(X, A)$.

The definition of cellular $G$-cohomology is given in [7] for the case of $\mathscr{F}=\{$ all subgroups of $G\}$. Recalling it we define the cellular $G$-(co) homology with coefficient in a $G$-coefficient system $\mathfrak{i}$ (or $\mathfrak{m}$ ) for an orbit type family $\mathscr{F}$ as follows.

Let $(X, A)$ be a pair of $G$-CW complexes; in addition, suppose that $X$ is a $G-\mathrm{CW}_{\mathscr{F}}$ complex, that is by definition, $X$ is a $G$-CW complex such that $G_{\sigma} \in \mathscr{F}$ for every (closed) $G$-cells $G \sigma$ of $X$. We remark that this is equivalent to say the $G$-coefficient system is generic with respect to $X$. The module $C_{p}^{G, c}(X, A ; \mathfrak{l})$ of cellular $G$-chains with coefficient in $\mathfrak{f}$ is defined by

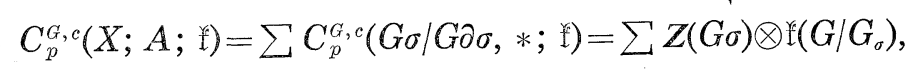

where the summations range over all (closed) $G$-p-cells $G \sigma$ of $X-A$. As is

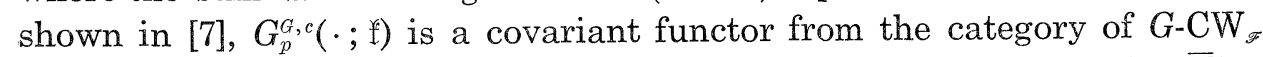
complexes and $G$-cellular maps to the category of abelian groups. Let $\bar{X}^{i}=$ $X^{i} \cup A$. Then, there is a canonical isomorphism

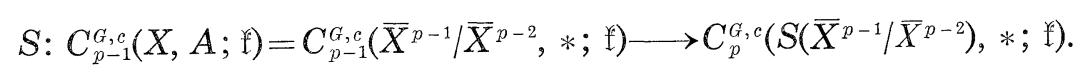

The boundary homomorphism $\partial^{c}: G_{p}^{G, c}(X, A ; \mathfrak{l}) \rightarrow C_{p-1}^{G, c}(X, A ; \mathfrak{l})$ is defined by $\partial^{c}=C_{p}^{G, c}(\partial) \circ S$, where $\partial$ is the boundary map of the horizontal Puppe sequence in the following homotopy commutative diagram:

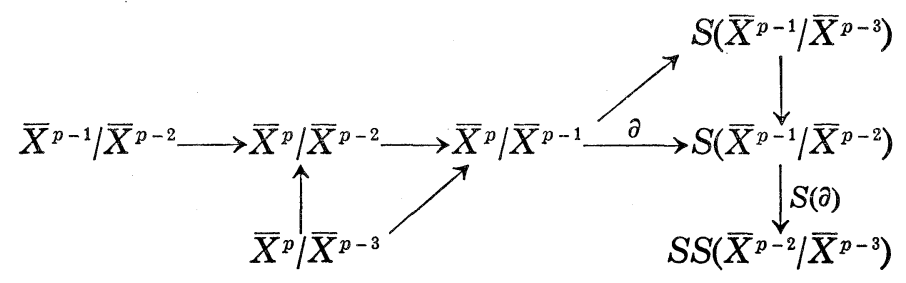


Note that $C_{p}^{G, c}(S(\partial) \circ \partial)$ depends only on the $G$-homotopy class of

$$
S(\partial) \circ \partial: \bar{X}^{p} / \bar{X}^{p-1} \longrightarrow S S\left(\bar{X}^{p-2} / \bar{X}^{p-3}\right) .
$$

Then, $\partial^{c} \circ \partial^{c}=C_{p}^{G, c}(S(\partial) \circ \partial) \circ S \circ S=0$, because both the vertical and oblique sequences are cofiberings and hence $S(\partial) \circ \partial$ is $G$-homotopic to the trivial map. The homology module $H_{p}\left(C_{*}^{G, c}(X, A ; \mathfrak{l}), \partial^{c}\right)$ is called a cellular $G$ homology of $(X, A)$ with coefficient in $\mathfrak{i}$ and denoted by $H_{p}^{G, c}(X, A ; \mathfrak{f})$.

We define the module $C_{G}^{p, c}(X, A ; \mathfrak{m})$ of cellular $G$-cochains of $(X, A)$ with coefficient in $\mathrm{m}$ by

$$
\begin{gathered}
C_{G}^{p, c}(X, A ; \mathfrak{m})=\left\{c^{*}: C_{p}^{G, c}\left(X, A ; Z_{\mathscr{F}}\right) \longrightarrow \mathfrak{m}(G / H),\right. \text { homomorphisms } \\
\text { with } \left.c^{*}(G \sigma) \in \mathfrak{m}\left(G / G_{\sigma}\right)\right\} .
\end{gathered}
$$

Then, $G_{G}^{p, c}(X, A ; \mathfrak{m})=\prod C_{G}^{p, c}(G \sigma / G \partial \sigma, * ; \mathfrak{m})$ where the product ranges over all (closed) $G$-p-cells $G \sigma$ of $X-A$, and $C_{G}^{p, c}(\cdot ; \mathfrak{m})$ is a contravariant functor from the category of $G$-CW $\mathrm{CW}_{\mathscr{F}}$ complexes and $G$-cellular maps to the category of abelian groups. The coboundary homomorphism is defined by $\delta^{c}=C_{G}^{p, c}(\partial ; \mathfrak{m})$ $\circ S^{*}$, where

$$
S^{*}: C_{G}^{p-1, c}(X, A ; \mathfrak{m})=C_{G}^{p-1, c}\left(\bar{X}^{p-1} / \bar{X}^{p-2}, * ; \mathfrak{m}\right) \longrightarrow C_{G}^{p, c}\left(S\left(\bar{X}^{p-1} / \bar{X}^{p-2}\right), * ; \mathfrak{m}\right)
$$

is the canonical isomorphism. Then, $\delta^{c} \circ \delta^{c}=0$ is the also verified in the same way as in the proof of $\partial^{c} \circ \partial^{c}=0$. The cohomology module $H^{p}\left(C_{G}^{*, c}(X\right.$, $\left.A ; \mathfrak{m}), \delta^{c}\right)$ is called a cellular $G$-cohomology of $(X, A)$ with coefficient in $\mathfrak{m}$ and denoted by $H_{G}^{p, c}(X, A ; \mathfrak{m})$.

The exactness and dimension axioms are satisfied for the cellular $G$-(co)homology functor. Also, the excision axiom, expressing that the inclusion $(Y, Y \cup A) \smile(Y \cup A, A)$ induces an isomorphism, follows directly from the definition. The homotopy axiom is verified by the $G$-cellular approximation theorem (Proposition 0.2) as in [7].

Let now $\mathscr{F}=\{$ all subgroups of $G\}$. If we consider $H_{*}^{G, c}\left(K_{X}, K_{A} ; \mathfrak{f}\right)$ and $H_{G}^{*, c}\left(K_{X}, K_{A} ; \mathfrak{m}\right)$ as functors defined on a pair $(X, A)$ of $G$-spaces, they are $G$-homology and $G$-cohomology theories satisfying all seven equivariant Eilenberg-Steenrod axioms (cf. [4]). In fact, the seven axioms except homotopy and excision axioms are easily verified. For the homotopy axiom we use Remark following the proof of Theorem 1. For the excision axiom it suffices to show that $K_{X-U} \cap K_{A}=K_{A-U}$ and $K_{X-U} \cup K_{A} \longrightarrow K_{X}$ is a $G$-homotopy equivalence provided that Int $A \supset \bar{U}$. But we do not give the proof here, because we shall demonstrate Proposition 3.1 and Theorem 3 by using only the seven axioms and the additive property for the singular $G$-(co) homology of Ilman and the definition of the cellular $G$-(co) homology. 
Proof of Proposition 3.1 for $G$-Homology. We put

$$
H(p, q)=\sum H_{n}^{G}\left(\bar{X}^{1-p}, \bar{X}^{1-q} ; \mathfrak{f}\right) \quad\left(\bar{X}^{i}=X^{i} \cup A ; X^{\infty}=X, X^{i}=\phi(-\infty \leqq i<0)\right) .
$$

Then, by the properties of the singular $G$-homology given in [4], we see that the collection of $H(p, q)$ satisfies the axioms (SP. 1-5) of Cartan-Eilenberg $[2$, p. 334] and hence induces a spectral sequence

$$
\left(\bar{E}_{r}^{p, q}, \bar{d}_{r}^{p, q}\right)=\left(E_{-p,-q}^{r}, d_{-p,-q}^{r}\right)
$$

It is clear that $E_{p, q}^{1}=H_{p+q}^{G}\left(\bar{X}^{p}, \bar{X}^{p-1} ; \mathfrak{f}\right)$. Since

$$
\left(\bar{X}^{p}, \bar{X}^{p-1}\right) \smile\left(\bar{X}^{p} \cup C \bar{X}^{p-1}, C \bar{X}^{p-1}\right) \longrightarrow\left(\bar{X}^{p} / \bar{X}^{p-1}, *\right)
$$

are an excision map and a $G$-homotopy equivalence (by the $G$-homotopy extension property), we can identify as

$$
\begin{aligned}
E_{p, q}^{1} & =H_{p+q}^{G}\left(\bar{X}^{p}, \bar{X}^{p-1} ; \mathfrak{f}\right)=H_{p+q}^{G}\left(\bar{X}^{p} / \bar{X}^{p-1}, * ; \mathfrak{f}\right)=\sum H_{p+q}^{G}(G \sigma / G \partial \sigma, * ; \mathfrak{f}) \\
& =\sum H_{q}^{G}\left(G / G_{\sigma} ; \mathfrak{l}\right)=\sum Z(G \sigma) \otimes \mathfrak{f}_{q}\left(G / G_{\sigma}\right)=C_{p}^{G, c}(X, A ; \mathfrak{f})
\end{aligned}
$$

by the additivity property of the singular $G$-homology, where the summations range over all (closed) $G$-p-cells $G \sigma$ of $X-A$ and

$$
G \sigma / G \partial \sigma=\left(G / G_{\sigma} \times \Delta^{p}\right) /\left(G / G_{\sigma} \times \partial \Delta^{p}\right)=S^{p}\left(G / G_{\sigma} \cup *\right) .
$$

Furthermore, the 1st differential

$$
d_{p, q}^{1}: E_{p, q}^{1}=C_{p}^{G, c}\left(X, A ; \mathfrak{f}_{q}\right) \longrightarrow E_{p-1, q}^{1}=C_{p-1}^{G, c}\left(X, A ; \mathfrak{f}_{q}\right)
$$

coincides with the boundary homomorphism $\partial^{c}$ in the chain complex of cellular $G$-chains of $(X, A)$, because they both send $G \sigma \otimes a$ to

$$
\sum_{\tau} \sum_{g_{\lambda(\sigma, \tau)}}\left[\sigma, g_{\lambda(\sigma, \tau)} \tau\right] G \tau \otimes \mathfrak{f}_{q}\left(\hat{g}_{\lambda(\sigma, \tau)}\right)(a) \text {. }
$$

Here, we refer the reader to [7] for the notation; $\tau$ ranges over the representatives of all (closed) $G$ - $(p-1)$-cells and $g_{\lambda(\sigma, \tau)}$ ranges over all the arcwise connected components of $\left(G / G_{\tau}\right)^{G_{\sigma}}$. When $G_{\sigma} \subset G_{g \tau}, \hat{g} ; G / G_{\sigma} \rightarrow G / G_{\tau}$ is defined by $\hat{g}\left(h G_{\sigma}\right)=h g G_{\tau}$ and $[\sigma, g \tau]$ denotes the degree of the map

$$
\begin{aligned}
S^{p} & =\sigma / \partial \sigma \subset(G \sigma / G \partial \sigma)^{G_{\sigma}} \subset\left(\bar{X}^{p} / \bar{X}^{p-1}\right)^{G_{\sigma}} \stackrel{\partial}{\longrightarrow} S\left(\bar{X}^{p-1} / \bar{X}^{p-2}\right)^{G_{\sigma}} \\
& =S(\vee G \tau / G \partial \tau)^{G_{\sigma}} \longrightarrow S(G \tau / G \partial \tau)^{G_{\sigma}} \\
& =S\left(\vee g_{\lambda(\sigma, \tau)} \tau / g_{\lambda(\sigma, \tau)} \partial \tau\right) \longrightarrow S(g \tau / g \partial \tau)=S^{p}
\end{aligned}
$$

Therefore,

$$
E_{p, q}^{2}=H_{p}^{G, c}\left(X, A ; \mathfrak{\mathfrak { f }}_{q}\right)
$$


Note that $H_{n}^{G}\left(X, \bar{X}^{p+1} ; \mathfrak{f}_{q}\right)=0$ for $n \leqq p+1$, because $H_{n}^{G}\left(\bar{X}^{s}, \bar{X}^{s-1} ; \mathfrak{f}_{q}\right)=0$ for $n \leqq s-1$. So, $j_{*}: H_{p}^{G}\left(\bar{X}^{p+1}, \bar{X}^{p-1} ; \mathfrak{f}_{q}\right) \rightarrow H_{p}^{G}\left(X, \bar{X}^{p-1} ; \mathfrak{f}_{q}\right)$ induced by the inclusion $j$ is an isomorphism. This means that the spectral sequence is weakly convergent. Also, $X^{-1}=\phi$ implies that $F^{1} H=0$, and so it is strongly convergent.

q.e.d.

Proof of Theorem 3 for G-homology. We notice that $\mathfrak{f}_{q}$ in Proposition 3.1 satisfies $\mathfrak{f}_{0} \mid \mathscr{F}=\mathfrak{f}$ and $\mathfrak{f}_{q}(G / H)=H_{q}^{G}(G / H ; \mathfrak{l})=0$ for $H \in \mathscr{F}$ and $q \neq 0$ by the dimension axiom [4]. So, since the isotropy subgroups of $K_{X}$ are contained in $\mathscr{F}$, the spectral sequence $E_{p, q}^{r}$ for $\left(K_{X}, K_{A}\right)$ instead of $(X, A)$ given in the above proof satisfies $E_{p, q}^{2}=0$ for $q \neq 0$. In particular, it collapses and $H_{p}^{G, c}\left(K_{X}, K_{A} ; \mathfrak{l}\right)=E_{p, 0}^{2}=E_{p, 0}^{\infty}=H_{p}^{G}\left(K_{X}, K_{A} ; \mathfrak{f}\right)$ which coincides with $H_{p}^{G}(X, A ; \mathfrak{l})$ by Theorem 2 .

q.e.d.

To prove Proposition 3.1 for $G$-cohomology we prepare the following lemma.

Lemma 3.2. $H_{G}^{n}\left(X, X^{p+1} \cup A ; \mathfrak{m}\right)=0$ for $n \leqq p+1$ for any contravariant G-coefficient system $\mathrm{m}$.

Proof. Let $Y$ denote the $G$-CW complex $X /\left(X^{p+1} \cup A\right)$. Note that $H_{G}^{n}\left(X, X^{p+1} \cup A ; \mathfrak{m}\right) \cong H_{G}^{n}(Y ; *: \mathfrak{m})$ and there is an exact sequence (cf. Proof of Lemma 2.5):

$$
0 \rightarrow \lim { }^{1} H_{G}^{n-1}\left(Y^{k}, * ; \mathfrak{m}\right) \rightarrow H_{G}^{n}(Y, * ; \mathfrak{m}) \rightarrow \lim H_{G}^{n}\left(Y^{k}, * ; \mathfrak{m}\right) \rightarrow 0 .
$$

So, it suffices to show $H_{G}^{q}\left(Y^{k}, Y^{p+1} ; \mathfrak{m}\right)=0$ for any $k<\infty$ and $q \leqq p+1$ because $Y^{p+1}=*$. But, this follows from the fact that

$$
\begin{aligned}
H_{G}^{q}\left(Y^{\ell}, Y^{\ell-1} ; \mathfrak{m}\right) & =H_{G}^{q}\left(Y^{\ell} / Y^{\ell-1}, * ; \mathfrak{m}\right)=\prod H_{G}^{q}(G \sigma / G \partial \sigma, * ; \mathfrak{m}) \\
& =\prod H_{G}^{q-\ell}\left(G / G_{\sigma} ; \mathfrak{m}\right)=0 \quad \text { when } \quad q-\ell<0 . \quad \text { q.e.d. }
\end{aligned}
$$

Proof of Proposition 3.1 for G-cohomology. The proof is almost the same. Put $H(p, q)=\sum H_{G}^{n}\left(\bar{X}^{q-1}, \bar{X}^{p-1} ; \mathfrak{m}\right)$ and let $\left(E_{r}^{p, q}, d_{r}^{p, q}\right)$ denote the associated spectral sequence. Then, we can identify as

$$
\begin{aligned}
E_{1}^{p, q} & =H_{G}^{p+q}\left(\bar{X}^{p}, \bar{X}^{p-1} ; \mathfrak{m}\right)=H_{G}^{p+q}\left(\bar{X}^{p} / \bar{X}^{p-1}, * ; \mathfrak{m}\right) \\
& =\prod H_{G}^{p+q}(G \sigma / G \partial \sigma, * ; \mathfrak{m})=\prod H_{G}^{q}\left(G / G_{\sigma} ; \mathfrak{m}\right)=C_{G}^{p, c}\left(X, A ; \mathfrak{m}^{q}\right),
\end{aligned}
$$

where the products range over all (closed) $G-p$-cells $G \sigma$ of $X-A$. And

$$
\begin{aligned}
d_{1}^{p, q} & =\delta^{c}: E_{1}^{p, q}=H_{G}^{p+q}\left(\bar{X}^{p}, \bar{X}^{p-1} ; \mathfrak{m}\right)=C_{G}^{p, c}\left(X, A ; \mathfrak{m}^{q}\right) \rightarrow E_{1}^{p+1, q} \\
& =H_{G}^{p+q+1}\left(\bar{X}^{p+1}, \bar{X}^{p} ; \mathfrak{m}\right)=C_{G}^{p+1, c}\left(X, A ; \mathfrak{m}^{q}\right),
\end{aligned}
$$

because 


$$
\left(d_{1}^{p, q} c^{*}\right)(G \sigma)=\sum_{\tau} \sum_{g_{\lambda(\sigma, \tau)}}\left[\sigma, g_{\lambda(\sigma, \tau)} \tau\right] \mathfrak{m}^{q}\left(\hat{g}_{\lambda(\sigma, \tau)}\right) c^{*}(G \tau)=\left(\delta^{c} c^{*}\right)(G \sigma) .
$$

Here, the notation and summation are the same as in the case of $G$-homology. So,

$$
E_{2}^{p, q}=H_{G}^{p, c}\left(X, A ; \mathfrak{m}^{q}\right) .
$$

By Lemma 3.2 we see that the inclusion induces an isomorphism

$$
H_{G}^{p}\left(X, \bar{X}^{p-1} ; \mathfrak{m}^{q}\right) \longrightarrow H_{G}^{p}\left(\bar{X}^{p+1}, \bar{X}^{p-1} ; \mathfrak{m}^{q}\right),
$$

which implies that the spectral sequence is weakly convergent. Moreover, $H_{G}^{n}\left(X, \bar{X}^{p} ; \mathfrak{m}^{q}\right)=0$ for $n \leqq p$ implies $\cap F^{p} H=0$, that is, the spectral sequence is strongly convergent.

q.e.d.

Proof of Theorem 3 for G-cohomology. We apply Proposition 3.1 to $\left(K_{X}, K_{A}\right)$ (instead of $\left.(X, A)\right)$. Then, $\mathfrak{m}^{0} \mid \mathscr{F}=\mathfrak{m}$ and $\mathfrak{m}^{q}(G / H)=H_{G}^{q}(G / H ; \mathfrak{m})=0$ for $H \in \mathscr{F}$ and $q \neq 0$ by the dimension axiom for $H_{G}^{*}$. So, since $K_{X}$ is a $G-\mathrm{CW}_{\mathscr{T}}$ complex, $E_{2}^{p, q}=0(q \neq 0)$ and $H_{G}^{p, c}\left(K_{X}, K_{A} ; \mathfrak{m}\right)=E_{2}^{p, 0}=E_{\infty}^{p, 0}=H_{G}^{p}\left(K_{X}, K_{A} ; \mathfrak{m}\right)$, which coincides with $H_{G}^{p}(X, A ; \mathfrak{m})$ by Theorem 2 .

q.e.d.

Added In Proof: The author thanks Professor P. May who informed him of the paper "R.M. Seymour: Some functorial constructions on G-spaces, Bull. London Math. Soc., 15(1983), 353-359" which treated also the G-CW approximation of the $G$-space when $G$ is a compact Lie group.

\section{References}

[1] E. Brown, Abstract homotopy theory, Trans. Amer. Math. Soc., 119 (1965), 79-85.

[2] H. Cartan and S. Eilenberg, Homological Algebra, Princeton Univ. Press, Princeton, 1956.

[ 3 ] H. Hastings and S. Waner, On Brown's construction for compact Lie group actions, Preprint.

[4] S. Illman, Equivariant singular homology and cohomology I, Mem. Amer. Math. Soc., 156 (1975), 1-74.

[5] T. Matumoto, Equivariant $K$-theory and Fredholm operators, J. Fac. Sci. Univ. Tokyo Sect. IA, 18 (1971), 109-125.

[6] T. Matumoto, On G-CW complexes and a theorem of J. H. C. Whitehead, J. Fac. Sci. Univ. Tokyo Sect. IA, 18 (1971), 363-374.

[7] T. Matumoto, Equivariant cohomology theories on G-CW complexes, Osaka J. Math., 10 (1973), 51-68.

[ 8 ] T. Matumoto, N. Minami and M. Sugawara, On the set of free homotopy classes and Brown's construction, Hiroshima Math. J., 14 (1984), 359-369.

[ 9 ] J. Milnor, On axiomatic homology theory, Pacific J. Math., 12 (1962), 337-341.

[10] D. Montogomery and L. Zippin, Topological Transformation Groups, Interscience Publ., New York, 1955. 
[11] M. Murayama, On G-ANR's and their G-homotopy type, Osaka J. Math., 20 (1983), 479-512.

[12] R. Switzer, Algebraic Topology-Homotopy and Homology, Springer-Verlag, Berlin, 1975.

[13] S. Waner, Equivariant homotopy theory and Milnor's theorem, Trans. Amer. Math. Soc., 258 (1980), 351-368.

\author{
Department of MATHEMAtics \\ FACULTY OF SCIENCE \\ HiROSHIMA UNIVERSITY \\ HIROSHIMA 730, JAPAN
}

